\title{
$4 \mathrm{mad} 9 \mathrm{ge} 9(20.2 \mathrm{ramm}$ Der Internist
}

Organ des Berufsverbandes Deutșcher Internisten

$$
\sqrt{27}-8 \sqrt{0}
$$

Jahrgang 29, 1988

Begründet von

G. Budelmann $\cdot H$. von Kress $\cdot H$. Reinwein W. Ruge $\cdot H$. Schwiegk $\cdot F$. Valentin

Unter Mitwirkung von
H. Weinholz $\cdot$ E. Wetzels $\cdot$ E. Schüller
R. Schindlbeck

(Für den Vorstand des Berufsverbandes Deutscher Internisten)

R. Aschenbrenner $\cdot$ H.E. Bock - M. Broglie

V. Harth - W. Hoffmeister - W. Rick

Herausgegeben von

E. Buchborn, München - M. Classen, München W. Dölle, Tübingen · R. Gross, Köln J. van de Loo, Münster · G. Riecker, München P.C. Scriba, Lübeck · W. Siegenthaler, Zürich P. von Wichert, Marburg

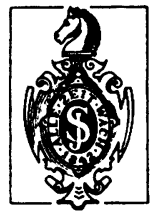

Springer-Verlag Berlin Heidelberg NewYork London Paris Tokyo 
pnoe unauffällig. Im Röntgenbild des Thorax zeigte sich eine zunächst vorwiegend interstitielle Pneumonie, die im Verlauf dann

\title{
Sehstörungen bei einem 42jährigen Mann mit erworbenem Immundefizit-Syndrom (AIDS)
}

\author{
M.M. Ritter ${ }^{1}$, B. Jacob ${ }^{1}$, D. Jüngst ${ }^{1}$ und V. Klauss ${ }^{2}$ \\ ${ }^{1}$ Medizinische Klinik II (Direktor: Prof. Dr. G. Paumgartner) \\ des Klinikums Großhadern \\ ${ }^{2}$ Augenklinik (Direktor: Prof. Dr. O.-E. Lund) der Universität München
}

Bei einem 41jährigen homosexuellen Mann wurde während eines stationären Aufenthaltes wegen einer Pneumocystis-carinii Pneumonie die Diagnose eines erworbenen ImmundefizitSyndroms (AIDS) gestellt. Ein Jahr später traten erstmals Sehstörungen auf. In der folgenden Arbeit werden die ophthalmologischen Befunde im Verlauf dargestellt und über den Erfolg einer experimentellen Therapie berichtet.

\section{Fallbeschreibung}

Ein 41jähriger homosexueller Mann wurde wegen seit fünf Wochen zunehmender Dyspnoe und seit zwei Wochen bestehendem Fieber bis zu $40^{\circ} \mathrm{Celsius} \mathrm{stationär} \mathrm{eingewie-}$ sen. Wesentliche Vorerkrankungen bestanden nicht. Ein Jahr vor der Aufnahme war eine orale Candidiasis ambulant erfolgreich behandelt worden. Der körperliche Untersuchungsbefund war bis auf eine Tachykardie und eine zentrale Zyanose mit Orthoauch eine intraalveoläre Exsudation aufwies und am vierten Tage zur Intubation führte. Sowohl in einer Bronchiallavage als auch in einer transbronchialen Biopsie konnte das Protozoon Pneumocystis-carinii nachgewiesen werden und somit die Diagnose ,erworbenes Immundefizit-Syndrom" gesichert werden. Unter einer Therapie mit Trimethoprim (TMP) und Sulfamethoxazol (SMZ) in einer Tagesdosis von 20 bzw. $100 \mathrm{mg}$ pro kg Körpergewicht entfieberte der Patient und konnte nach vier Tagen wieder extubiert werden. Einen Monat später wurde er in sehr gutem Allgemeinzustand mit einer Rezidivprophylaxe von 5 bzw. $25 \mathrm{mg}$ TMP und SMZ pro kg und Tag entlassen, die jedoch nach einem halben Jahr wegen gastrointestinaler Nebenwirkungen vorübergehend abgesetzt werden mußte. Abgesehen von einer sofort empirisch mit TMP und SMZ behandelten und weniger schwer verlaufenden erneuten Pneumonie etwa $1 \mathrm{Jahr}$ später ist der Patient bis heute-zwei Jahre nach Diagnosestellung - weitgehend beschwerdefrei und körperlich voll belastbar. 
Antikörper gegen HIV ließen sich nachweisen, ferner bestand eine zelluläre Anergie und ein im Verlauf weitgehend konstantes, extrem erniedrigtes OKT $4 / 8$ Verhältnis von 0,03 . Die Luesserologie zeigte eine sogenannte Seronarbe, gegen Toxoplasmose, Zytomegalie und Epstein-Barr-Virus ließen sich Durchseuchungstiter nachweisen.

\section{Ophthalmologische Befunde}

Ein Jahr nach Diagnosestellung berichtete der Patient von einer Gesichtsfeldeinschränkung am rechten Auge. Nachdem eine ophthalmologische Untersuchung ein halbes Jahr zuvor einen unauffälligen Augenhintergrund gezeigt hatte, fanden sich jetzt erhebliche Veränderungen an beiden Augen: Als Ursache der Sehstörung konnte ein frisches entzündliches Infiltrat im Bereich der rechten Macula nachgewiesen werden. Daneben fanden sich große Infiltrate der nasalen Netzhautperipherie des linken und der temporalen des rechten Auges. Die zentralen Netzhautabschnitte des linken Auges wiesen zu diesem Zeitpunkt lediglich vier Cotton-wool-spots auf. Wir boten dem Patienten eine (experimentelle) Therapie mit dem Acyclovir-Derivat Dihydroxypropoxymethylguanosin (DHPG) an, er lehnte diese jedoch auf Grund seines guten Allgemeinzustandes und aus Furcht vor möglichen Nebenwirkungen $\mathrm{ab}$.

In der Folgezeit bereiteten sich die Entzündungsherde ,,steppenbrandförmig“ aus. Fünf Monate nach der ersten Augen-Symptomatik war der Patient auf dem rechten Auge völlig erblindet, und der Augenhinter- grund zeigte beidseits das Bild einer Panretinitis. Am linken Auge war allein die Macula von dem entzündlichen Prozeß ausgespart, so daß dem Patienten ein Visus von 0,5 erhalten blieb (Abb. 1). Da nun eine komplette Erblindung innerhalb kürzester Zeit vorausgesagt werden konnte, stimmte der Patient der Therapie mit DHPG zu.

Gemäß dem Therapieprotokoll wurden $2 \times 5 \mathrm{mg}$ pro $\mathrm{kg}$ Körpergewicht und Tag für einen Zeitraum von drei Wochen gegeben. Die entzündlichen Infiltrate kamen nach zwei Wochen zum Stillstand, flackerten jedoch zwei Wochen nach Beendigung der Therapie wieder auf. Daraufhin wurde erneut mit derselben Dosis therapiert und anschließend mit einer Dauertherapie von $5 \mathrm{mg}$ pro $\mathrm{kg}$ für fünf Tage pro Woche begonnen. Der Stillstand der Entzündung ist für das linke Auge in der Abbildung 2 dokumentiert. Dieses Bild ist unter der Dauertherapie mittlerweile sieben Monate unverändert, der Visus auf dem linken Auge beträgt 0,4 .

Ein Zytomegalie-Virus-Nachweis im Urin oder Rachenspülwasser gelang nicht, ebensowenig fand sich ein Titeranstieg in der Serologie. Ein kraniales Computer- und Kernspintomogramm waren unauffällig. Das typische ophthalmologische Bild, der Verlauf, das Ansprechen auf DHPG und das Rezidiv nach Absetzen sicherten die Diagnose.

\section{Diagnose}

Erworbenes - Immundefizit - Syndrom (AIDS) mit Z.n. Pneumocystis-carinii Pneumonie und Zytomegalie-Retinitis.

\section{Diskussion}

Fünf Arbeitsgruppen haben systematisch die ophthalmologischen Befunde bei Patienten mit AIDS untersucht und darüber berichtet (Tabelle 1) $[3,4,6$, 7, 11]. Eine sechste Gruppe berichtet über ihre autoptisch gewonnenen Ergebnisse (Tabelle 1) [9].

Als häufigster Befund am Augenhintergrund finden sich bei etwa der Hälfte der Patienten Cotton-wool-spots, wie sie auch von anderen Erkrankungen bekannt sind. Sie sind komplett rückbildungsfähig, was sich auch im Verlauf des hier vorgestellen Patienten zeigte. Ebenfalls rückbildungsfähig sind die häufig in der Umgebung der Cottonwool-spots auftretenden retinalen $\mathrm{Hä-}$ morrhagien (oder sog. Roth-spots).

Der zweithäufigste Befund am Augenhintergrund ist die durch ZytomegalieViren hervorgerufene Entzündung von Ader- und Netzhaut, von der etwa jeder fünfte der lebenden und jeder dritte der verstorbenen Patienten mit AIDS betroffen ist. Das entzündliche Ödem der Retina und die Infiltration des Glaskörpers verhindern den freien Durchblick auf die darunter liegende rötliche Aderhaut, so daß die Infiltrationen weißlich-gelb imponieren. Die unscharfe Begrenzung gibt den frischen Entzündungsherden ein flauschiges Aussehen, oftmals kommen Hämorrhagien hinzu (Abb. 1). Die bevorzugte
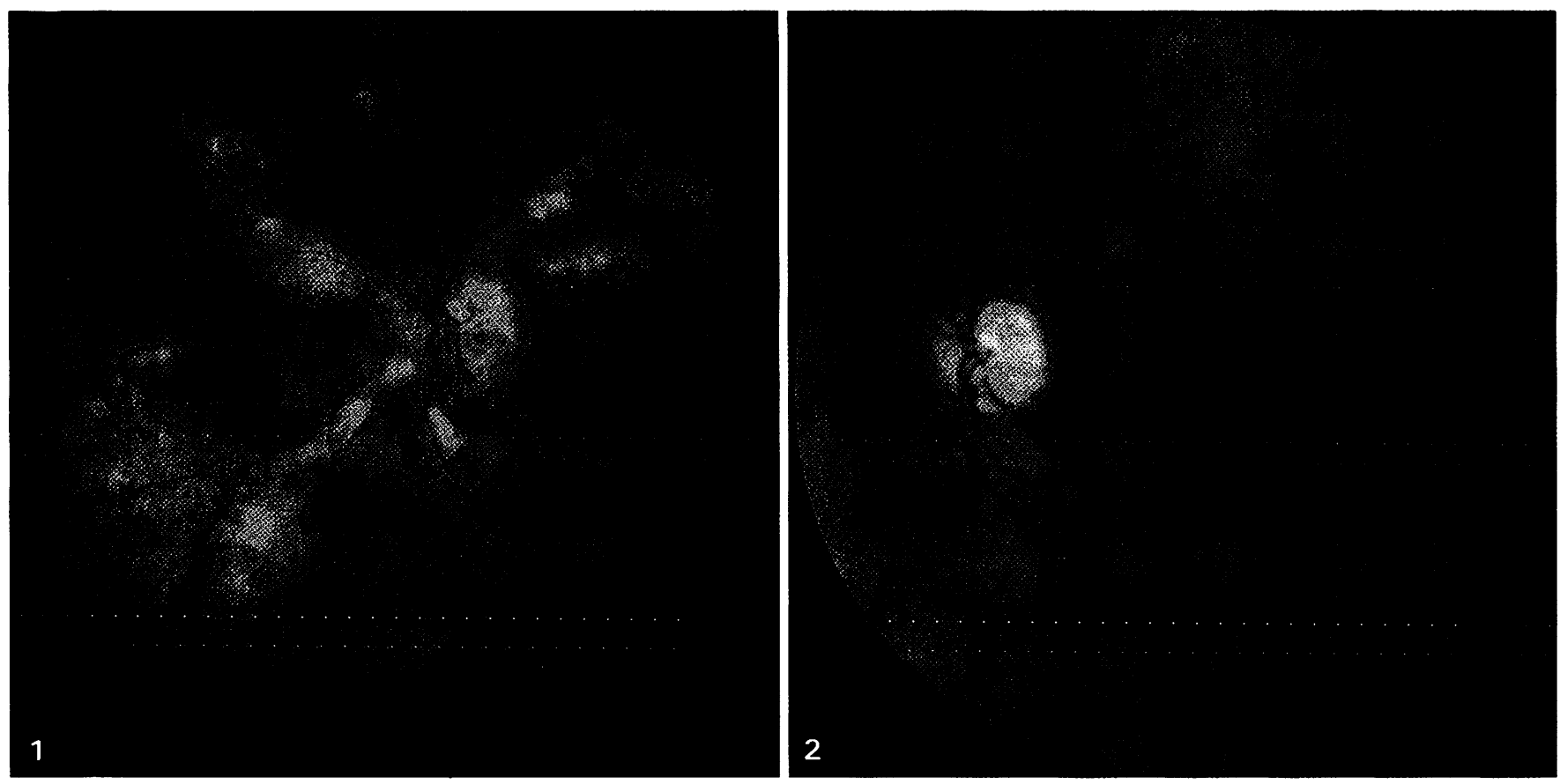

Abb. 1. Panretinitis des linken Auges fünf Monate nach der ersten Augensymptomatik

Abb. 2. Weitgehende Abheilung der Entzündung unter DHPD-Therapie 
Tabelle 1. Ophthalmologische Befunde bei Patienten mit AIDS

$N \quad$ CWS Re. Hä. CMV Path. Bcfunde
insgesamt (\%)

Holland et al. [4] Rosenberg et al. [11] Khadem et al. [6] Palestine et al. [7] Freeman et al. [3]

Pepose et al. [9]

(Autopsien)

$(\mathrm{CWS}=$ Cotton wool spots; $\mathrm{Re} . \mathrm{Hä}=$ retinale Hämorrhagien $; \mathrm{CMV}=$ Zytomegalic Chorioretinitis)

Ausbreitung entlang der Netzhautgefäße und die typische entzündliche Beteiligung der Gefäßwände sind ebenfalls gut erkennbar (Abb. 1). Die Ausbreitung der Retinitis wird als „,steppenbrandartig" charakterisiert; die Bezeichnung rührt daher, daß die frischesten Infiltrate sich häufig am Übergang des schon betroffenen Retina-Abschnittes zur noch gesunden Retina finden, während die dahinter liegenden Abschnitte bereits narbig verheilen (Abb. 1). Die Pigmentblattdegenerationen führen schließlich zum körnigen Aussehen des Augenhintergrundes (Abb. 1).

Der charakteristische Ablauf und die typische Morphologie der CMV-Retinitis sind mehrfach betont worden [1, 5-8]. Da andere opportunistische Infektionen des Auges ( z.B. durch Toxoplasma gondii, Kryptokokken, Mycobacterium avium-intracellulare oder Pneumocystis carinii) sehr selten sind $[3,4,7,9,11]$ und sich dafür weder klinisch noch bei den technischen Untersuchungen (CT, NMR, Serologie) ein Anhaltspunkt ergab, entschlossen wir uns trotz fehlendem Virusnachweis bei drohender Erblindung zur Therapie. Die insgesamt zweimal negativen Kulturen auf Zytomegalie-Virus im Urin und Rachenspülwasser sprechen nur gegen die hier auch klinisch nicht erkennbare Generalisation der Erkrankung. Ein Titeranstieg eines Durchseuchungstiters wird auf Grund der gestörten humoralen Abwehr oftmals (auch bei anderen Infektionen) bei Patienten mit AIDS nicht beobachtet.

Weitere vereinzelt beobachtete ophthalmologische Befunde bei Patienten mit AIDS sind Augenmotilitätsstörungen und Kaposi-Sarkome [4, 7].

\section{Therapie}

Zur Therapie von Infektionen mit humanpathogenen Herpesviridae (zu die- ser Gruppe gehört neben dem Zytomegalie-Virus noch das Varizellen-ZosterVirus, das Epstein-Barr- und das Herpes-Simplex-Virus) stand bislang lediglich das Acyclovir zur Verfügung, das seine virostatische Wirksamkeit durch strukturelle Ähnlichkeit mit dem Desoxyguanosin erreicht.

Da eine für die Aktivierung von Acyclovir notwendige Kinase nur von den mit Varizellen-Zoster und Herpessimplex-Viren betroffenen Zellen gebildet wird, ist Acyclovir nur gegen diese beiden Herpesviridae-Viren therapeutisch sinnvoll einsetzbar, gegen CMV und EBV aber wirkungslos [2].

Das sich in der klinischen Erprobung befindliche Acyclovir-Derivat Dihydroxypropoxymethylguanosin (DHPG) kann hier nun eine therapeutische Lücke schließen, da es gegen alle humanpathogenen Herpesviridae wirksam ist. Der Wirkungsmechanismus ist nicht letztlich geklärt, kommt aber möglicherweise über eine Bindung an die virale DNA-Polymerase zustande [10].

Von wenigen Einzelberichten abgesehen, haben bisher drei Gruppen über ihre Erfahrungen mit der Therapie einer Chorioretinitis bei Patienten mit AIDS berichtet $[5,8,10]$. Regelhaft führt die Anwendung dieses Medikamentes zu einer Besserung oder zu einem Stillstand der Entzündung, aber leider kommt es mit gleicher Regelmäßigkeit - wie es auch bei dem hier vorgestellten Patienten beobachtet wurde - nach dem Absetzen der Therapie zu einem Rezidiv der Erkrankung. Die häufigste Nebenwirkung ist die Granulozytopenie, die wir in noch tolerablem Ausmaß auch bei unserem Patienten beobachteten und die bei etwa jedem 10. Patienten zum Abbruch der Therapie zwang. Seltener kommt es zu makulopapulösen Exanthemen oder Transaminasenerhöhungen.

\section{Resumee}

Dieser Fallbericht unterstreicht einmal mehr, wie wichtig die Zusammenarbeit aller Fachrichtungen bei der Betreuung der Patienten mit erworbenem Immundefizit-Syndrom ist. Hierzu gehört auch die sorgfältige ophthalmologische Untersuchung. Er soll auch demonstrieren, daß die Zytomegale - Chorioretinitis unbehandelt zur Erblindung führt (wie hier am rechten Auge geschehen) und nicht - wie einige erste Berichte vermuten ließen - ausschließlich im Finalstadium des AIDS auftritt. Die experimentelle Therapie mit DHPG sollte in jedem Einzelfall erwogen werden und muß nach den bisherigen Erfahrungen als Dauertherapie lebenslang fortgeführt werden.

Danksagung. DHPG wurde von Syntex research in England zur Verfügung gestellt.

\section{Literatur}

1. D’Amico DJ, Talamo JH, Felsenstein D, Hirsch MS, Albert DM, Schooley RT (1986) Ophthalmoscopic and histologic findings in cytomegalovirus retinitis treated with BW-B759U. Arch Ophthalmol 104:1788-1793

2. Elion GB (1982) Mechanism of action and selectivity of acyclovir. Am J Med $73: 7-13$

3. Freeman WR, Lerner CW, Mines JA, Lash RS, Nadel AJ, Starr MB, Tapper ML (1984) A prospective study of the ophthalmologic findings in the acquired immune deficiency syndrome. Am J Ophthalmol 97: 133-142.

4. Holland GN, Pepose JS, Pettit TH, Gottlicb MS, Yec RD, Foos RY (1983) Acquired immune deficiency syndrome - ocular manifestations. Ophthalmology 90:859-873

5. Holland GN, Sakamoto MJ, Hardy D, Sidikaro Y, Kreiger AE, Frenkel LM (1986) Treatment of cytomegalovirus retinopathy in patients with acquired immunodeficiency syndrome. Arch Ophthalmol 104:1794-1800

6. Khadem M, Kalish SB, Goldsmith JA, Fetkenhour C, O'Grady RB, Phair JP, Chrobak M (1984) Ophthalmologic findings in acquired immune deficiency syndrome (AIDS). Arch Ophthalmol 102:201-206

7. Palestine AG, Rodrigues MM, Macher AM, Chan CC, Lane HC, Fauci AS, Masur H, Longo D, Reichert CM, Steis R, Rook AH, Nussenblatt RB (1984) Ophthalmic involvement in acquired immunodeficiency syndrome. Ophthalmology $91: 1092-1099$

8. Palestine AG, Stevens G, Lane HC, Masur H, Fujikawa LS, Nussenblatt RB. Rook AH, Manischewitz J, Baird B, Megill M, Quinnan G, Gelmann E, Fauci AS (1986) Treatment of cytomegalovirus 
retinitis with dihydroxypropoxymethyl guanine. Am J Ophthalmol 101:95 101 9. Pepose JS, Holland GN, Nestor MS, Cochran AJ, Foos RY (1985) Acquired immune deficiency syndrome - pathogenic mechanisms of ocular disease. Ophthalmology $92: 472-484$

10. Rosecan LR, Stahl-Bayliss CM, Kalman
CM, Laskin OL (1986) Antiviral therapy for cytomegalovirus retinitis in AIDS with dihydroxypropoxymethyl guanine. Am J Ophthalmol 101:405-418

11. Rosenberg PR, Uliss AE, Friedland GH Harris CA, Small CB, Klein RS (1983) Acquired immunodeficiency syndrome ophthalmic manifestations in ambula- tory patients. Ophthalmology $90: 874$ 878

Dr. M.M. Ritter

Medizinische Klinik II

Klinikum Großhadern

Marchioninistraße 15

D-8000 München 70 


\section{Sachregister}

Die Seitenzahlen in Klammern bezeichnen Buchbesprechungen

Acetylsalicylsäure, Embryotoxisches Risiko 196

Aciclovir, Embryotoxisches Risiko 202

ACTH-Resistenz, Rezeptorstörung 447

Adematosis coli, Gardner-Syndrom, Kasuistik 699

Adipositas, Hypertonie arterielle 274

Adrenozeptor-Effektor-System, Kopplungsmechanismen 399

Adrenozeptoren, kardialc, Bedeutung 409

Adrenozeptoren, Lokalisation, Funktion 389

AIDS, Neurologische Manifestationen (651)

AIDS-Patient, Sehstörungen, Kasuistik 590

AIDS-Patienten, HIV-infizierte Patienten 92

AIDS-Phobic, Symptomatologie 99

AIDS-Vorfeldpatienten, Drogenabhängigkeit 99

AIDS-Vorfcldpatienten, Homosexualität 98

Akupunktur, Akupunkturpunkte 480

Akupunktur, Anwendungsmöglichkeiten 483

Akupunktur, Diagnostische Differenzierung 481

Akupunktur, Entsprechungssystem Yin und Yang 480

Akupunktur, Indikationen nach WHO 485

Akupunktur, Meridian-System 481

Akupunktur, Wirkprinzip 479

Akupunktur, 5-Funktionskreise 483

Aldosteronismus primärer, Kasuistik 697

Alkohol, Hypertonie 273

Alkoholgenuß, Gefäßprotektion 836

Alkoholgenuß, Kulturelle Faktoren 325

Alkoholismus, Definitionskriterien 301

Alkoholismus, Diagnostische Verfahren 302

Alkoholismus, Diagnostische Verfahren, Entscheidungskriterien 305

Alkoholismus, Elterlicher Einfluß 326

Alkoholismus, Fragebogentests 302

Alkoholismus, Häuligkeit Bundesrepublik 323

Alkoholismus, Laboruntersuchungen 303

Alkoholismus, Prävention, Administrative Maßnahmen 326

Alkoholismus, Prävention, Individuelle Faktoren 325

Alkoholismus, Repräsentativbefragungen 323

Alkoholismus, Therapie, Behandlungserfolge 316

Alkoholismus, Therapie, Behandlungskette 313

Alkoholismus, Therapie, Behandlungsmethoden 314

Alkoholismus, Therapie, Behandlungsziele 313

Alkoholismus, Therapie, Entwöhnungsphase 315

Alkoholismus, Therapie, Entzugsphase 314
Alkoholismus, Therapie, Entzugssyndrom, Distraneurin 315

Alkoholismus, Therapie, Kontaktphase 314

Alkoholmißbrauch, Alkohol-Syndrom fetales 335

Alkoholmißbrauch, cocarzinogene Wirkung 335

Alkoholmißbrauch, Medikamenteninteraktionen 335

Alkoholmißbrauch, Organschäden, Bronchopummonale 331

Alkoholmißbrauch, Organschäden, Endokrinium 330

Alkoholmißbrauch, Organschäden, Gastrointestinaltrakt 332

Alkoholmißbrauch, Organschäden, Hämolytische Anämien 331

Alkoholmißbrauch, Organschäden, Herz 329

Alkoholmißbrauch, Organschäden, Hypertonie 330

Alkoholmißbrauch, Organschäden, Leber 333

Alkoholmißbrauch, Organschäden, Nervensystem 334

Alkoholmißbrauch, Organschäden, Pankreas 333

Alkoholmißbrauch, Organschäden, Rhabdomyolyse 331

Alkoholmißbrauch, Organschäden, Schlaganfall 330

Alkoholmißbrauch, Persönlichkeitsveränderungen, Objektivierungstests 309

Alkoholmißbrauch, Psychische Leistungsfähigkeit 308

Alkoholmißbrauch, Psychische Leistungsfähigkeit, Gedächtnis 309

Alkoholmißbrauch, Psychische Leistungsfähigkeit, Motorik 309

Alkoholmißbrauch, Psychische Veränderungen 307

Alkoholmißbrauch, Psychische Veränderungen, Objektivierung 310

Alkoholmißbrauch, Psychosyndrom organisches 308

Alkohol-Psychosen, Alkohol-Delir 307

Alkohol-Psychosen, Alkohol-Halluzinose 308

Alkohol-Psychosen, Korsakow-Syndrom 308

Alkoholstoffwechsel, Alkoholdehydrogenase 318

Alkoholstoffwechsel, Äthanoloxidation, Lipidstoffwechsel 319

Alkoholstoffwechsel, Äthanoloxidation, Methabolische Konsequenzen 319

Alkoholstoffwechsel, Äthanoloxidation, Mikrosomale Enzyminduktion 319

Alkoholstoffwechsel, Äthanolstoffwechsel 317

Alkoholstoffwechsel, Mirkosomales oxidierendes System 318

Alkoholwirkung, beim Menschen (102)

Alpha-Adrenozeptor, Veränderungen, Herzinsuffizienz 407
Alpha-Adrenozeptor, Veränderungen, $\mathrm{Hy}$ pertonie 404

Alpha-Adrenozeptoren, Nachweis 399

Alpha-Adrenozeptoren, Regulationsmechanismen 401

Alpha-Adrenozeptoren, Subtypen 397

Analgeticaabusus, Hämolytische Anämien 365

Analgeticanephropathie 365

Androgenresistenz, Rezeptorstörung 450

Angiologie, Atlas (346)

Angiologie, Kompaktwissen (777)

Angiologie praktische (726)

Angst-Syndrome, Benzodiazepin-Rezeptor 425

Angst-Syndrome, Gamma-Aminobuttersäure 425

Antacida, Ulcustherapie 735

Antiarrhythmica, Herzrhythmusstörungen, Verstärkung 338

Antiarrhythmica, proarrhythmischer Effekt 338

Antiarrhythmica, proarrhythmischer Effekt, Bedeutung 343

Antiarrhythmica, proarrhythmischer Effekt, Begünstigende Faktoren 341

Antiarrhythmica, proarrhythmischer Effekt, Elcktrophysiologie 342

Antiarrhythmica, proarrhythmischer Effekt, Tachykardien 338

Antiarrythmica, proarrhythmischer Effekt Torsade de pointes 339

Antibiotica, Fehlanwendung, Resistenzentwicklung 345

Antihypertensiva, Niereninsuffizienz, Dosisanpassung 242

Antirheumatica, nicht steroidale, Embryotoxisches Risiko 200

Antithrombin III-Mangel, angeborener, Komplikationen, Kasuistik 54

Antithrombin III-Mangel, Ursachen 55

Arterielle Verschlußkrankheit, Therapie, Alternative Methoden 501

Arterielle Verschlußkrankheit, Therapie, Hämatogene Oxydation (HOT) 501

Arteriographie selektive, Pankreascyste, Kasuistik 587

Arzneibehandlung moderne (306)

Arzneimittel, Bioverfügbarkeit, PlasmaKonzentrations-Zeitkurve 182

Arzneimittel, Neben- und Wechselwirkungen (12)

Arzneimittel, Neben- und Wechselwirkungen (57)

Arzneimittel, Pharmakokinetik, Plasma-Eiweiß-Bindung 188

Arzneimittel, Pharmakokinetik, Speziesvariation 184

Arzneimittel, Plazentarübertragung 186

Arzneimittel, Schwangerschaft, Teratogene Substanzen, Reaktionsmechanismen 632

Arzneimittel, Teratogene Wirkung, Prinzipien 631

Arzneimittel, Teratogene Wirkung, Substanzgruppen, Übersicht 634 
Arzneimittel, Teratogene Wirkung, Übersicht 634

Arzneimittel, Teratogenität, Pharmakokinetische Grundlagen 179

Arzneimittel, Teratogenität, Plazentarübertragung 186

Arzneimittel, Teratogenität, Spezies-Abhängigkeit 181

Arzneimittel, Tcratogenität, Spezies-Unterschiede 180

Arzneimittel, Teratogenität, Thalidomid 183

Arzneimittel, Teratogenität, Untersuchungsmethoden 179

Arzneimittel, Teratogenität, Arzneimittelmetabolismus 182

Arzneimittelallergie, Allergie-Phasen 161

Arzneimittelallergie, Allergische Reaktion, Immungenetischer Einfluß 166

Arzneimittelallergie, Allergische Reaktion, Immunogenität 165

Arzneimittelallergie, Allergische Reaktion, Individuelle Faktoren 168

Arzneimittelallergie, Allergische Reaktion, Klinische Manifestation, Übersicht 164

Arzneimittelallergie, Allergische Reaktion, Ursachen 165

Arzneimittelallergie, Allergische Reaktionen Typ IV, Immunreaktive Lymphozyten 163

Arzneimittelallergie, Allergische Reaktionen Typ I-III, Antikörperbildung 161

Arzneimittelallergie, Arzneimittel, Allergisierendes Potential 161

Arzneimittelallergie, Immunantwort, TLymphozyten 165

Arzneimittelallergic, Immunogenität, Proteinbindung 163

Arzneimittelallergie, Immunpharmakalogische Grundlagen 160

Arzneimittelgebrauch, Klare Indikationsstellung 142

Arzneimittelgebrauch, Magisches Adjuvans 141

Arzneimittelgebrauch, Naturheilkunde 146

Arzneimittelgebrauch, Orgasnismus als Reagenz 142

Arzneimittelgebrauch, Risikobeurteilung 143

Arzneimittelgebrauch, Risikobeurteilung 145

Arzneimittelschäden, Risikobeurteilung 145

Arzneimittelwirkung, Teratogene Potenz 631

Arzneiverordnungen (549)

Arzt als Arznei (576)

Ärztliches Berufsrecht (216)

Ärztliches Handeln, Empirie 459

Ärztliches Handeln, Empirische Behandlung, Indikationen 461

Ärztliches Handeln, Wissenschaftliche Erfahrung 460

Aspergillose, allergische bronchopulmonale, Kasuistik 832

Aspergillose, bronchopulmonale, Diagnose-Kriterien 834

Aspergillose, Respirationstrakt, Antikörperklassen 833

Asthma bronchiale, Beta-Rezeptoren-Antikörper 441
Asthma bronchiale, Beta-Rezeptoren-Veränderungen, Inflammatorische 441

Asthma bronchiale, Bronchialsystem, Adrenerge Rezeptoren 440

Asthma bronchiale, Bronchialsystem, Cholinerge Rezeptoren 439

Asthma bronchiale, Calmodulin (297)

Asthmaforschung (462)

Atemregulationsstörungen, Pulmonale Hypertonie 681

Atmungsgefährdung. Schlaf (576)

Autonomes Adenom der Schilddrüse, Szintigraphie 542

Balneologie (169)

Bartter-Syndrom, Rezeptorstörung 452

Behcet-Syndrom, Diagnosekriterien 357

Benzodiazepin-Rezeptor 427

Beta-Adrenozeptor, Vcränderungen, Herzinsuffizienz. 407

Beta-Adrenozeptor, Vcränderungen, Hypertonie 404

Beta-Adrenozeptoren, Nachweis 399

Beta-Adrenozeptoren, Regulationsmechanismen 402

Beta-Adrenozeptoren, Subtypen 398

Beta-Blockerbehandlung, Beta-Adrenozeptor-Dichte, kadiale 403

Bilio-enterale Fistelbildung, Cholelithiasis, Kasuistik 51

Bioäquivalenz,, in-vitro-Äquivalenzstandard 154

Bioäquivalenz, Serumkonzentrations-Zeitkurve 153

Bioäquivalenz, Untersuchungsmethoden 152

Bleiintoxikation chronische, Kasuistik 648

Bleivergiftungen, Organmanifestationen 650

Bodybuilding, Myoglobinuric 456

Bronchialmuskulatur, Cholinerg-adrenerges Gleichgewicht, Inflammatorische Einflüsse 439

Bronchialmuskulatur, Cholinerge Innervation 438

Bronchialmuskulatur, Sympathische Innervation 438

Bronchialzirkulation, Anatomie 664

Bronchialzirkulation, Bronchien, Blutversorgung 665

Bronchialzirkulation, Bronchopulmonale Anastomosen 665

Bronchialzirkulation, Hypervaskularisation 667

Bronchialzirkulation, PEEP-Beatmung 667

Bronchialzirkulation, Pulmonale Hypertonie 664

Bronchitis chronisch obstruktive, Pulmonale Zirkulation 671

Bronchus, Blutversorgung 665

Calcitonin, Nasale Applikation 298

Campylobacter-pylori-induzierte akute Gastritis, Therapie 747

Campylobacter-pylori-induzierte Chron Typ B-Gastritis, Therapie 748

Campylobacter-pylori-positives Ulcus duodeni, Therapie 751

Campylobacter-pylori-positives Ulcus ventriculi, Therapie 751
Carcinoid-Syndrom, metastasierendes, Kasuistik 347

Carcinomschmerzen, Opiatanalgesie (544)

Carotis-Endarteriektomie, Indikation 140

Cerebrovasculäre Insulfizienz, Gingko biliboa 470

Chemotherapic (611)

Chincsische Medizin, Akupunktur 505

Chinesische Medizin, Arzneidrogen 505

Chinesische Medizin, Europäische Verbreitung 506

Chinesische Medizin, Gesundheitsbegriff 504

Chinesische Medizin, Grundzüge 504

Chinesische Medizin, Wissenschaftlichkeit 508

Cholangiolithiasis, Bchandlungskonzept 796

Cholangiolithiasis, Behandlungskonzept 799

Cholangiolithiasis, Biliäre Pankreatitis, Therapie 797

Cholangiolithiasis, Endoskopische Extraktion, erfolglose, Therapic 797

Cholangiolithiasis, Endoskopische Therapie 797

Cholangiolithiasis, Stoßwellenlithotrypsie, extrakorporale 794

Cholangiolithiasis, Therapie, Ergebnisse 796

Cholecysto-gastrische Fistel, Kasuistik 589

Cholecystolithiasis, Litholyse percutantranshepatische, Ergebnisse 789

Cholecystolithiasis, Litholyse percutantranshepatische, Methyl-tert-ButylÄther 788

Cholecystolithiasis, Litholyse percutantranshepatische, Perspektiven 790

Cholecystolithiasis, Litholyse percutantranshepatische, Technik 788

Cholecystolithiasis, Litholyse percutantranshepatische, Voraussetzungen 788

Cholecystolithiasis, Litholyse-Therapic, Kosten 790

Cholecystolithiasis, Stoßwellenlithotrypsic, extrakorporale 793

Cholelithiasis, Bilio-enterale Fistelbildung Kasuistik 51

Cholelithiasis, Stoßwellen-Lithotripsie, extrakorporale 792

Colitis ischämische, postoperative, Kasuistik (289)

Colitis ischämische, Therapie 290

Colon, Gefäßversorgung 290

Coloncarcinom, Schwangerschaft 623

Colorektales Carcinom, Haemoccult-Screening (139)

Coloskopic, Komplikationen 816

Coloskopie, Parasitendiagnostik 810

Conn-Syndrom, Kasuistik 697

Cor pulmonale, Therapie symptomatische 693

Cor pulmonale, Ursachen 688

Co-Trimazol, Bioäquivalenzstudien 155

Crohnsche Krankheit, Diagnostik, Kasuistik 285

Crohnsche Krankheit, Polyarthritis, Kasuistik 285

Cumarin-Nekrose, Antithrombin III-Mangel, angeborener, Kasuistik 54

Cushing-Syndrom, Cortisol, Funktionstests 255 
Cushing-Syndrom, Diagnostik 255

Cushing-Syndrom, Pathophysiologie 254

Cushing-Syndrom zentrales, Transphenoidale Hypophysenoperation, Kasuistik 636

Cyclosporintherapie. Graft-versus-hostReaktion 836

Cytomegalievirus-Infektionen, Nierentransplantation, Symptomatik 362

Cytostaticatherapic maligner Erkrankungen (486)

Delirium tremens, Klinik 307

Demenz im Alter (663)

Depressive Syndrome, Serotonin-Hypothese 422

Diabetes insupidus, renaler, Rezeptorstörung 446

Diabetes mellitus. Gestationsdiabetes 607

Diabetes mellitus. Herz-K reislauferk rankungen (12)

Diabetes mellitus, Insulintherapie (297)

Diabetes mellitus, Ratgeber (429)

Diabetes mellitus, Schwangerschaft 606

Diabetes mellitus, Stresshormone (471)

Diabetes mellitus Typ II b, Pathophysiologie 394

Diabetische Nephropathie, Hypertoniebehandlung 241

Diabetische Nephropathic, Progredienz. Hypertoniebedeutung 241

Diagnoseschlüssel ncurologischer der WHO (509)

Dialysebehandlung, Schwangerschaft 604

Dialysepatient, Hypertonic. Dialyseverfahren 244

Dialysepatient, Hypertonicbehandlung 244

Diäletik, K rebserk rankungen, Einflüsse 492

Diätetik, Makrobiotische Nahrung, Vilamin B12 594

Diuretica, Pharmakologie (544)

Dumping-Syndrom, Phytotherapic 466

Echinacca-Präparate, Immunstimulation 476

Echinokokkus, Endoskopische Diagnostik 811

Enddarmerkrankungen, Ärztlicher Rat (62)

Endokrine Unterfunktion, Mögliche Ursachen 445

Endoskopie, Gastroenterologie (455)

Endoskopie gastrointestinale, Komplikationen, Todesfälle 815

Endoskopie, Oberer Verdauungstrakt, EKG-Veränderungen 817

Endoskopie, Oberer Verdauungstrakt. Mortalität 816

Enterale Sondenernährung, endoskopische Zugänge, Vorteilc 801

Enteralc Sondenernährung, Komplikationen 801

Enterocolitis, Polyarthritis, Assoziation 287

Eosinophiles Lungengranulom, Histiozytosis X, Kasuistik 639

EPH-Gestose, Gerinnungsstörung 616

EPH-Gestose, HELLP-Syndrom, Kasuistik 354

ERCP, Komplikationen 817

ERCP, Komplikationen, Pankreatitis 817
Erfahrungsmedizin 459

Ernährungslchre, Grundbegriffe (486)

Erythropoeitin, Klinische Anwendung 719

Fieberschübe intermittierende, Lyme-Borreliose, Kasuistik 780

Fruchtwasserembolic, Gerinnungsstörung 615

Fruchtwasserembolic, Therapic 616

Gardner-Syndrom, Kasuistik 699

Gastroenterologie internistische (231)

Gastrointestinalblutung obere, Blutstillung, Endoskopische Methoden 756

Gastrointestinalblutung obere, Blutungslokalisationen, Letalität 755

Gastrointestinalblutung obere, Blutungsursachen 755

Gastrointestinalblutung obere, Endoskopische Therapie, gesicherte Verfahren 755

Gastrointestinalblutung obere, Nichtvarizenblutung, Endoskopische Therapieverfahren 760

Gastrointestinalblutung obere, Nichtvarizenblutung, Lasertherapie 760

Gastrointestinalblutung obere, Notfallendoskopie, Bedeutung 756

Gastrointestinalblutung obere, Stadieneinteilung nach Forrest 759

Gastrointestinale Dysfunktion, Phytotherapie 464

Gastrointestinaltrakt, Funktionelle Störungen (532)

Gastroskopie, Komplikationen 815

Gastrostomiesonde, endoskopisch plazierte. Enterale Ernährung 802

Geburtenkontrolle. WHO-Impistoff 652

Generika, Bioäquivalenz 147

Generika, Bioäquivalenz. 149

Generika, Bioäquivalenz, Therapeutische Bedeutung 150

Gencrika. Bioäquivalenz, Verglcichsstudien 155

Generika, Bioäquivalenzstudien, Ergebnisse 158

Generika, Bioverfügbarkeit 149

Generika, Kritische Bioverfügbarkeit 151

Gencrika, Therapeutische Äquivalenz 149

Generika, Verordnungstrend 148

Geriatrische Medizin (81)

Gerinnungsstörungen, Geburtshilfe, Diagnostik 612

Gerinnungsstörungen, Geburtshilfe, Krankheitsbilder 613

Gesundheitspolitik (139)

Gingko biliboa, Durchblutungsförderung 470

Glibenclamid, Bioäquivalenzstudien 156

Glomerulonephritiden, Schwangerschaft, Prognose 603

Glomerulonephritis, Hypertonie renale, Übersicht 240

Gold-Therapic, Gold-Nephropathie, Spontanrückbildung 220

Gonadotropin-Rcsistenz, Rezeptorstörung 448

Goodpasture-Syndrom, Augenveränderungen, Kasuistik 704

Granulozyten-Koloniestimulierender Faktor, G-CSF, Klinische Anwendung 718

Granulozyten-Makrophagen-Koloniesti- mulierender Faktor GM-CSF, Klinische Anwendung 718

Gyrasehemmer, Antibakterielles Spektrum 207

Gyrasehemmer, Anwendungsgebiete 210

Gyrasehemmer, Chinoline 206

Gyrasehemmer, Niereninsuffizienz 211

Gyrasehemmer, Pharmakokinetik 209

Gyrasehemmer, Resistenzentwicklung 209

Gyraschemmer, Wirkungsmechanismus 206

Haarzellenleukämie, Therapie, Interferonalpha 720

Hacmoccult-Screening, Colorektales Carcinom (139)

Hämanginom, Leber, Fettinfarkte, Kasuistik 291

Hämatologie klinische (593)

Hämatopoetische Wachstumsfaktoren, Klinische Anwendung 718

Hämobilic, Pankreascyste, Kasuistik 586

Hämolyse intravasale, Ferrokinetik 783

Hämolyse intravasale, mechanische, Herzklappenersatz, Kasuistik 781

Harnsteinanalyse, Infrarotspektroskopie, Ergebnisse 820

Harnsteinanalyse, Konkrementanalyse, Infrarotspektroskopic 818

HDL, Cholesterin-Transport, LipoproteinStoffwechsel 379

HDL-bindende Rezeptoren 380

HDL-Zellinteraktion 380

Heparintherapie, Thrombozylopenie, Kasuistik 515

Hepatologie (519)

Herz, Elektrophysiologic (306)

Herzinfarkt akuter, Thrombolysetherapie (368)

Herzinsulfizienz., Adrenorezeptorveränderungen 407

Herzklappenersatz, Hämolyse mechanische, Kasuistik 781

Herzkrankhcit hypertensive, Therapic 265

Herzmuskelhypertrophic, hypertensive, linksventrikuläre konzentrische 261

Herzrhythmusstörungen, Antiarrhythmica, proarrhythmischer Effekt 338

Herzrhythmusstörungen, Ventrikuläre Arrhythmien, Antiarrhythmica-induzierte Verstärkung 340

Herztod plötzlicher (312)

Herztod plötzlicher, Kasuistik 350

Histamin-H2-Antagonisten, Ulcustherapie 734

Histiozytosis X, Krankheitsbilder, Überblick 640

HIV-assoziierte Krankheiten, Diagnostik und Therapie 82

HIV-assoziierte Krankhciten, Generalisierte Lymphadenopathie, Klinik 82

HIV-assoziierte Krankheiten, HIV-Infektion akutc, Klinik 82

HIV-assoziierte Krankheiten, Klassifikation, Allgemeinsymptome 83

HIV-assoziierte Krankheiten, Neoplasien, Kaposi-Syndrom 88

HIV-assoziierte Krankheiten, Neoplasien, Maligne Lymphome 88

HIV-assoziierte Krankheiten, Nervensystem 89

HIV-assoziierte Krankhciten, Opportunistische Infektionen, Candida-Oesophagitis 85 
HIV-assoziierte Krankheiten, Opportunistische Infektionen, Herpesvirus-Infektionen 86

HIV-assoziierte Krankheiten, Opportunistische Infektionen, Kryptosporidiosen 87

HIV-assoziierte Krankheiten, Opportunistische Infektionen, Mykobakteriosen 87

HIV-assoziierte Krankheiten, Opportunistische Infektionen, Pneumocystis carinii-Pneumonie 83

HIV-Infektion, Blutspendewesen, AIDSAufklärungsbroschüre 124

HIV-Infektion, Blutspendewesen, Autologe Transfusion 129

HIV-Infektion, Blutspendewesen, Bluttransfusion 126

HIV-Infektion, Blutspendewesen, Prävention 128

HIV-Infektion, Blutspendewesen, Untersuchungsbogen 125

HIV-Infektion, Bluttransfusion, transfusionsassoziiertes AIDS 127

HIV-Infektion. Epidemiologie, AIDS-Verbreitung weltweit 119

HIV-Infektion, Epidemiologie, Drogenabhängige 116

HIV-Infektion, Epidemiologic, Homosexuelle 115

HIV-Infektion, Epidemiologic, Infektionsverlauf 112

HIV-Infektion, Epidemiologic, Infektiosität 118

HIV-Infektion, Epidemiologie, Inzidenz. 118

HIV-Infektion, Epidemiologic, Medizinisches Personal 117

HIV-Infektion, Epidemiologie, Prävalenz. i.d. Bevölkerung 118

HIV-Infektion, Epidemiologic, Prostitution 117

HIV-Infektion, Epidemiologic, Risikogruppen 115

HIV-Infektion, Epidemiologic, Sexualverhalten 114

HIV-Infektion, Epidemiologic, Übertragungswege 114

HIV-Infektion, Epidemiologie, Verbreitung weltweit 119

HIV-Infektion, Epidemiologische Modelle 121

HIV-Infektion, Infektionsverlauf, Inkubationszeit 112

HIV-Infektion, Infektionsverlauf, Serokonversion 112

HIV-Infektion, Ophthalmologische Bcfunde 591

HIV-Infektion, Therapie, Antivirale Medikamente 103

HIV-Infektion, Therapie, Antivirale Medikamente, Adsorptionshemmung 103

HIV-Infektion, Therapie, Antivirale Mcdikamente, Azidothymidin 105

HIV-Infektion, Therapie, Antivirale Medikamente, Hemmung der Transkription 104

HIV-Infektion, Therapie, Antiviralc Medikamente, Hemmung der Translation 106

HIV-Infektion, Therapie, Antivirale Medikamente, Interferone 107
HIV-Infektion, Therapie, Antivirale Medikamente, Penetrationshemmung 103

HIV-Infektion, Therapic, Antivirale Medikamente, Retrovir 105

HIV-Infektion, Therapie, Immunstimulantien 107

HIV-Infektion, Therapie, Vakzine 108

HIV-Infektionen, Krankheitsstadien 92

HIV-Infektionen, Problematik 65

HIV-Infektionsprophylaxe, Atemspende 136

HIV-Infektionsprophylaxe, Desinfektion 133

HIV-Infektionsprophylaxe, Hygieneregeln 135

HIV-Infektionsprophylaxe, Infektionsgefährdende Untersuchungen 134

HIV-Infektionsprophylaxe, Infektionswege 131

HIV-Infektionsprophylaxe, Infektionswege, Medizinisches Personal 132

HIV-Infektionsprophylaxe, Laborbereich, Sterilisation 136

HIV-Infektionsprophylaxe, Risikoarmes Verhalten 133

HIV-Infektionsprophylaxc, Stationärer Bcreich 136

HIV-infizierte Patienten, Ambulante Betreuung 92

HIV-infizierte Patienten, Ambulante Betreuung, Ärztliche Aufgaben 93

HIV-infizierte Patienten, Ambulante Betreuung, Contact tracing 95

HIV-infizierte Patienten, Ambulante Betreuung. Kontrolluntersuchungen 94

HIV-infizierte Patienten, Ambulante Betreuung. Kooperation 92

HIV-infizicrte Patienten, Ambulante Betreuung, Lebensführung 93

HIV-infizierte Patienten, Probleme infizierter Frauen 99

HIV-infizicrte Patienten, Psychosoziale AIDS-Versorgung 100

HIV-infizicrte Patienten, Psychosoziale Probleme 97

HIV-Serodiagnostik, Anti-HIV-Bestimmung 74

HIV-Serodiagnostik, Anti-HIV-Bestimmung, Monospezifische Enzymimmunoassays 74

HIV-Serodiagnostik, Anti-HIV-Bestimmung, Western Blots 75

HIV-Serodiagnostik, Anti-HIV-Screening 75

HIV-Serodiagnostik, HIV-Antigen-Bestimmung 75

HIV-Serodiagnostik, HIV-Zellkultur 75

HIV-Serodiagnostik, HIV-2-Infektion 79

HIV-Serodiagnostik, Plausibilität der Befunde, Bestätigungslabor 76

HIV-Serodiagnostik, Schweizer Konzept 77

HIV-Scrologic, Infektion, Verlaufsbeurteilung 78

HIV-Serologie, Infektionsfrühphase, Befunde 77

HIV-Virologie, Genomstruktur 67

HIV-Virologie, HIV-1, Replikationszy-

klus 104

HIV-Virologie, Provirale Phase 73

HIV-Virologie, Replikationszyklus 68

HIV-Virologie, Virus-Gruppen 67

HIV-Virologic, Virusmorphologic 68
HIV-Virologic, Zytopathischer Effekt 69

HIV-2-Infektion, Epidemiologic 120

Hochdruckherz, Myokardhypertrophie,

Pathogenese 260

Homöopathie, Arzncimittelherstellung,

Dosierung 488

Homöopathie, Arzneistoffwirkung, Hahnemann 487

Homöopathic, Innere Medizin, Therapiemöglichkeiten 489

Homöopathic, Klinische Medizin, Unterschiede 490

Homöopathie, Krankheitslehre 488

Homöopathie, Lehrbuch (491)

Homöopathic, Simileprinzip 487

Homöopathie, Symptomenlehre, Individualisierung 488

Homöopathic, Therapic, Wirksamkcitsnachweis 490

Hormonresistenz-Syndrome, Übersicht 445

Hyperaldosteronismus primärer, Diagnostik 252

Hyperaldosteronismus primärer, Hypertonic 253

Hyperaldosteronismus primärer, Klinik 252

Hyperaldosteronismus sekundärer. Diagnostik 253

Hyperaldostcronismus sekundärer,

Plasmareninaktivität 253

Hyperaldosteronismus sekundärer. Therapic 254

Hyperprolactinaemic, Symptomatik 44

Hypertensive Herzkrankheit. Angina pectoris 266

Hypertensive Herzkrankheit, Differentialtherapie 265

Hypertensive Herzkrankheit, Differentialtherapic, Herzinsuffizienz 267

Hypertensive Herzkrankheit, Differentialtherapie, Herzrhythmusstörungen 267

Hypertensive Herzkrankheit, Differentialtherapie, Linksherzhypertropic 266

Hypertensive Krisc, Phäochromozytom, Therapie 258

Hyperthyreose immunogene, Langzeitbehandlung 581

Hyperthyreose immunogene, Medikamentöse Therapie 555

Hyperthyreose immunogene, Operative Therapie 556

Hyperthyreose immunogene, Radiojodtherapie 556

Hyperthyreose, Radiojodtherapie 578

Hyperthyreose, Szintigraphic 543

Hypertonie adrenale, Aldosteronismus primärer 252

Hypertonie adrenale, Aldosteronismus sekundärer 253

Hypertonie adrenale, Cushing-Syndrom 254

Hypertonie adrenale, Phäochromozytom 256

Hypertonie, Adrenorezeptorveränderungen, Organverteilung 404

Hypertonic arterielle, Definition 232

Hypertonic arterielle, Kardiale Manifestationen 260

Hypertonic arterielle, Schweregrade, Einteilung 224

Hypertonic arterielle, Therapic, Antihypertensiva 225 
Hypertonic artericlle, Therapie nicht medikamentöse, Ernährung 270

Hypertonie arterielle, Therapie nicht medikamentöse, Gewichtsreduktion 274

Hypertonie arterielle, Therapie, nichtmedikamentöse 225

Hypertonie milde, Definition 232

Hypertonic milde, Interventionsstudien, Antihypertensiva 236

Hypertonie milde, Interventionsstudien, Blutdrucksenkung 237

Hypertonie milde, Interventionsstudien, Frauen 236

Hypertonie milde, Interventionsstudien, Kardiovasculäre Vorerkrankungen, Therapiecrfolg 235

Hypertonic milde. Interventionsstudien, Therapieerfolg 234

Hypertonic mildc, Interventionsstudien. Übersicht 233

Hypertonic milde. Therapie. Interventionsstudien 233

Hypertonic renale. Niereninsuffizienz, Pathogenese 239

Hypertonie renovasculäre, Diagnostik, Angiographic 247

Hypertonie renovasculäre, Diagnostik, Captopril-Test 247

Hypcrtonie renovasculäre, Diagnostik, Nuklearmedizin 247

Hypcrlonic renovasculäre. Diagnostik. Screeningverfahren 246

Hypcrtonic renovasculäre, Diagnostik, Sonographic 246

Hypcrlonie renovasculäre, Diagnostik, Subtraktionsangiographie digitale 247

Hypertonie renovasculäre, Therapie, Katheterdilatation 248

Hypertonic renovasculäre, Therapic medikamentöse 248

Hypertonic renovasculäre, Therapic, Operationsverfahren 248

Hypcrionie. Schwangerschaftshochdruck 596

Hypertoniebehandlung, Compliance 279

Hypertoniebehandlung, Compliance, Behandlungsdauer 280

Hypcrtoniebehandlung. Compliance-bestimmende Faktoren 281

Hypertoniebehandlung, Compliance-Bcstimmung 279

Hypertoniebehandlung, Compliance-verbessernde Maßnahmen 282

Hypertoniebehandlung, Ernährung 270

Hypertoniebehandlung, Ernährung, Adipositas 274

Hypertoniebehandlung, Ernährung, Alkohol 273

Hypertonicbehandlung, Ernährung, Calcium 272

Hypertoniebehandlung, Ernährung, Eicosapentaensäure 274

Hypertonicbchandlung, Ernährung, Fette 274

Hypertoniebehandlung, Ernährung, Kalium 271

Hypertoniebehandlung, Ernährung, Kochsalz 270

Hypertonicbehandlung, Ernährung, Magnesium 272

Hypertoniebehandlung, Ernährung, Spurenelemente 273
Hypertoniebehandlung, Langzeittherapie, Drop-out-Rate 280

Hypertoniebehandlung, medikamentöse, Antihypertensiva 225

Hypertoniebehandlung, medikamentöse, Dosierung 229

Hypertoniebchandlung, medikamentöse. Pseudotherapicresistenz 228

Hypertoniebehandlung, medikamentöse, Stufentherapie 228

Hypertoniebehandlung, medikamentöse, Zweierkombination gezielte 227

Hypertoniebehandlung, medikamentöse, Zweierkombinationen 226

Hypertoniebehandlung, milde Hypertonie, Interventionsstudien 224

Hypertonicbchandlung, Niereninsuffizienz. Antihypertensiva 241

Hypertoniebehandlung, Therapie medikamentöse. Compliance 281

Hypoparathyreoidismus, Differentialdiagnose 448

Hypothyreose angeborene, Therapie 561

Hypothyreose, Langzeitbehandlung 582

Hypothyreose passagere, Therapie 561

Hypothyreose sekundäre, Therapie 562

Hypothyreose, Therapie 559

Hypothyreose, Therapie, Schilddrüsenpräparate 559

Hypoventilation, primäre alveoläre, Kasuistik 828

Hypoventilation, primäre alveoläre, Klinik 830

Hypoventilation, primäre alveoläre, Nosologie 830

Immunantwort, Endokrine Faktoren (663)

Immunpharmakologic (502)

Immunstimulation, Phytopräparatc, Applikationsweise 476

Immunstimulation, Phytopräparate, Arzneipflanzen 473

Immunstimulation, Phytopräparate, Echinacea-Präparate 475

Immunstimulation, Phytopräparate, Wirknachweis 473

Immunstimulation, Phytopräparatc, Wirkprinzipien 477

Infektionskrankheiten, Farbatlas (733)

Infektionskrankheiten, Schutzimpfungen (744)

Infrarotspcktroskopie, Konkrementanalysen 818

Infrarotspektroskopie, Methodik 818

Injektionstechniken (519)

Insulinresistenz, Parenterale Ernährung 803

Insulinresistenz, Pathophysiologie 391

Insulinrezeptor-Defekte, erworbene 393

Insulinrezeptor-Defekte, erworbene Störungen, Übersicht 394

Insulinrezeptor-Defekte, genetische 391

Insulinrezeptor-Defekte, genetische, Übersicht 392

Insulinrezeptor-Defckte, Krankheitsursachen 390

Intensivmedizin, Checklistc (629)

Intensivmedizin, Diagnostik und Therapic (744)

Interferon-alpha, Klinische Anwendung 720
Interferon-alpha, Klinische Anwendung, Nebenwirkungen 721

Interleukin 2, Klinische Anwendung 721

Internistische Therapic (744)

Isosorbiddinitrat, Bioäquivalenzstudien 156

Kalter Knoten, Operative Therapie 573

Kardiomyopathie dilatative, Virusmyokarditis 520

Kardiomyopathien, Alkoholkardiomyopathie 329

Kawasaki-Syndrom, Diagnosekriterien 357

Kernspin-Histotomograpie, hochauflösende 39

Kernspin-Spektroskopie, Blutplasma, Protonen NMR-Spektrum 35

Kernspin-Spektroskopie, Blutplasma, SpinRelaxationszeit 37

Kernspin-Spektroskopic, Kernspin-Prinzip 34

Kernspin-Tomographic, Protonensignale 38

Klinikseelsorge (696)

Knochenmarksnekrose, Leukämie akute 46

Kochsalzzufuhr, Hypertonie arterielle 270

Komatöse Zustände, Differentialdiagnose (102)

Konkrementanalysen, Infrarotspektroskopie 818

Konkrementanalysen, Infrarotspektroskopie, Analysengang 819

Konkrementanalysen, Infrarotspektroskopie, Ergebnisse 820

Kopfschmerz-Syndrome (429)

Koronare Herzkrankheit, Hypothyreose, Therapie 560

Koronare Herzkrankheit, Psychosoziale Faktoren (651)

Krankheitsbewältigung, hausärztliche

Krebs, im Alter (744)

Krebsdiäten 496

Krebserkrankungen, Diätetik 492

Krebserkrankungen, Diätetik, Anoxiehypothese, Warburg 493

Krebserkrankungen, Diätetik, Fette 494

Krebserkrankungen, Diätetik, Spezialkostformen 496

Krebserkrankungen, Diatetik, Vitamin C 495

Krebserkrankungen, Krebsdiäten 496

Krebspatient, Ernährung 497

Krebsregister, Organisationsformen 8

Kretinismus, Hypothyreose, TSH-Resistenz 447

Kryptokokkose, Diagnostik 512

Kryptokokkose, Lungenkryptokokkose, Kasuistik 510

Kryptokokkose, Therapie 512

Künstliche Ernährung, Enterale Sondenernährung, Lebensqualität 804

Künstliche Ernährung, Enterale Sondenernährung, Parenterale Ernährung 801

Laparoskopie, Parasitosen, Diagnostik 813

Lebertransplantation, Leberzirrhose primär biliäre 773

Lebertumoren benigne, Haemanginome 291 
Leberversagen akutes, Tumornekrosisfaktor 836

Leberzirrhose primär biliäre, Klinik 765

Leberzirrhose primär biliäre, Mangelernährung, Therapie 767

Leberzirrhose primär biliäre, Natürlicher Verlauf 765

Leberzirrhose primär biliäre, Osteopathie. Therapie 768

Leberzirrhose primär biliäre, Prognostische Faktoren 766

Leberzirrhose primär biliäre. Pruritus, Therapie 767

Leberzirrhose primär biliäre, Sicca-Symptomatik, Therapie 768

Leberzirrhose primär biliäre, Therapie, Azathioprin 769

Leberzirrhose primär biliäre. Therapie, Ciclosporin A 771

Leberzirrhose primär biliäre, Therapie, DPenicillamin 769

Leberzirrhose primär biliäre, Thcrapic, Lcbertransplantation 772

Leberzirrhose primär biliäre, Therapie, Prednisolon 770

Leberzirrhose primär biliäre, Therapie spezifische, Bewertungstabelle 772

Leberzirrhose primär biliäre, Therapie, Symptomatische Behandlung 766

Leberzirrhose primär biliäre, Therapie, Symptomatische Behandlung 766

Leberzirrhose primär biliäre, Therapie, $\mathrm{Ur}$ sodesoxycholsäure 771

Leukämie, akute, Knochenmarksnekrose 46

Leukämie akute, Knochenmarksnekrose, Kasuistik 46

Leukämie akute, Knochenmarksnekrose, Verlauf und Therapie 48

Leukämie, Chronisch myeloische, Kasuistik 645

Leukämie, chronisch myeloische. Therapic, Interferon-alpha 720

Lipoprotein-Rezcptoren, Übersicht 383

Lipoprotein-Stoffwechsel, CholesterinTransport, HDL 379

Lipoprotein-Stoffwechsel, ChylomikronenRezeptor 377

Lipoprotein-Stoffwechsel, HDL-Rezeptor 378

Lipoprotein-Stoffwechsel, HDL-Zellinteraktion 380

Lipoprotein-Stoffwechsel, LDL-Rezeptor 374

Lipoprotein-Stoffwechsel, LDL-Rezeptor, Bedeutung 376

Lipoprotein-Stoffwechsel, Lipoproteinrezeptoren 374

Lipoprotein-Stoffwechsel, Physiologic 373

Lipoprotein-Stoffwechsel, Plasmalipoproteine 372

Lipoprotein-Stoffwechsel, Rezeptorvermittelter, Übersicht 375

Lipoprotein-Stoffwechsel, Scavenger-Rezeptor 381

Lipoprotein-Stoffwechsel, VLDL-Rezeptor 382

Lungencarcinom (169)

Lungenemphysem, Antitrypsin-Mangel 673

Lungenemphysem, Blue bloater 673

Lungenemphysem, Pink puffer 673
Lungenerkrankungen interstitielle, Übersicht 676

Lungenerkrankungen obstruktive, Pulmonale Hypertonie, Therapieansat7. 688

Lungenerkrankungen, Röntgendiagnostik (139)

Lungenfibrose, Lungenbiopsie 677

Lungenfibrose, Prognose, Pulmonalarteriendruck 679

Lungenfibrose, Pulmonalc Hypertonic 677

Lungenfibrose, Pulmonale Hypertonie, Klinik 677

Lungenfibrose, Pulmonalc Hypertonie, Pathogenese 678

Lungentumoren (389)

Lungenversagen akutes (670)

Lyme-Erkrankunge, Intermittierende Fieberschübe, Kasuistik 778

Magnesiumsulfat, Eklampsie 599

Malaria, Diagnostik 60

Malaria, Prophylaxc 61

Malaria, Serologie 60

Malaria tropica, Kasuistik 59

Malaria tropica, Prognose 60

Malaria tropica, Therapie 60

Malignes Melanom. Schwangerschaft 624

Mamma-Carcinom, Pathogenese, Fettverzehr 494

Mammacarcinom, Schwangerschaft, Therapie und Verlauf 622

Marcumarnekrose 55

McDuffie's Syndrom, Kasuistik 824

Mediastinaltumoren, Thymus, Diagnostik 296

Medizinische Begutachtung (50)

Medizinische Erkenntnistheorie 593

Medizinische Ethik (33)

Minderwuchs, Clonidin, Therapie 64

Mißbildungen, Teratologie, Kritische Phase 170

Mißbildungsentstchung, Teratogene Medikamente, Dosicrung 632

Mißbildungsentstchung, Teratogene Medikamente, Fetale Gefährdung 631

Mißbildungsentstehung, Teratogene Medikamente, Plazentaschranke 632

Mißbildungsentstchung. Teratogene Medikamentenwirkung 630

Mißbildungsentstehung, Ursachen 630

Mitralinsuffizienz akute, Sehnenfadenruptur, Kasuistik 710

Monoklonale Antikörper, Klinische Relevanz (33)

Morbus Basedow, Operative Therapie 572

Myasthenia gravis, Autoantikörper, Pathogenese 416

Myasthenia gravis, Azetylcholinrezeptor, Autoantikörper 415

Myasthenia gravis, Azetylcholinrezeptor, Nachweis 415

Myasthenia gravis, Pathogenese 414

Myasthenia gravis, Pathogenese, Thymus 417

Myasthenia gravis, Rezeptorstörung, Antikörper-induzierte 416

Myasthenia gravis, Therapiemöglichkeiten 418

Myokardhypertrophie, Genetische Determination 264
Myokardhypertrophic, hypertensive 260

Myokardhypertrophie, hypertensive, Antihypertensive Therapie, Rückbildung 263

Myokardhypertrophie, hypertensive, linksventrikuläre exzentrische 262

Myokardhypertrophie, hypertensive, linksventrikuläre irreguläre 262

Myokardhypertrophie, hypertensive, Pathogenese 260

Myokardhypertrophie, hypertensive, Renin-Angiotensin-Aldosteron-System 264

Myokardhypertrophie, hypertensive,

Strukturveränderungen 265

Myokardhypertrophie, hypertensive, Sympathico-adrenerges-System 263

Myokardinfarkt, Akuttherapie, Plasminogen-Aktivator 652

Myxödemkoma, Therapic 561

Naturheilverfahren (368)

Nephrosklerose, Progredienz, Hypertoniebedeutung 241

Nephrotisches Syndrom, Urticaria, McDuffie's Syndrom 824

Neuronale Signalübertragung, Molekulare Grundlagen 420

Neurotransmitter, Depressive Syndrome 422

Neurotransmitter, Schizophrenie 424

Neurotransmitter, Überblick 420

Neurotransmitter-Rezeptoren, Signaltransduktion 421

Nierenerkrankungen, Hypertonic renalc 240

Nierenerkrankungen, Progredienz, Hypertoniebedeutung 240

Niereninsuffizienz, Hämolyse, Analgeticaabusus 365

Niereninsuffizienz, Hypertonicbchandlung, Antihypertensiva 241

Niereninsuffizienz, Hypertoniebchandlung, Antihypertensiva, Dosisanpassung 242

Niereninsuffizienz, Pharmakokinetik (368)

Nierenkrankheiten, in der Schwangerschaft, Prognose 603

Nierentransplantation, Immunsuppression, Cytomegalievirus-Infektion 360

Nierentransplantation, Immunsuppression, Gastroenteritis 360

Nierentransplantation, Schwangerschaft 604

Notfälle, Innere Medizin

Obstipations-Syndrom, Phytotherapie 468

Oesophagusvarizensklerosierung, Elektive Sklerosierung 758

Oesophagusvarizensklerosierung, Notfallsklerosierung 756

Oesophagusvarizensklerosierung, Prophylaktische Sklerosierung 758

Oesophagusvarizensklerosierung, Varizenokklusion 759

Onkogene, Tumorwachstum 432

Ophthalmopathie endokrine, Klassifizierung 526

Ophthalmopathie endokrine, Therapie 557

Osteomyelofibrose, Kasuistik 641

Osteoporose, Prophylaxe, Calcitonin nasales 298 
Ovarialcarcinom, Schwangerschaft, Therapic und Verlauf 621

Pancreatitis akute, Klinische Behandlung (306)

Pancreatitis chronische, Alkoholabusus 334

Papillomaviren (549)

Parasitosen biliäre, Endoskopische Diagnostik 813

Parasitosen gastro intestinale, Endoskopische Befunde 809

Parasitosen gastro intestinale, Klinische Symptomatik 807

Parasitosen gastro intestinale, Klinisches Bild 807

Parasitosen gastrointestinalc, Endoskopische Diagnostik 807

Parentcrale Ernährung, Enteralc Ernährung 801

Parenterale Ernährung, Komplikationen 801

Parenterale Ernährung, Nebenwirkungen, Cholestase 802

Parenterale Ernährung, Ncbenwirkungen, Funktionsverlust 802

Parenterale Ernährung, Nebenwirkungen, Insulinresistenz 803

Parenterale Ernährung, Nebenwirkungen, Leberverfettung 802

Pathophysiologie, klinische (322)

Pcriarteriitis nodosa, Pericarditis exsudaliva, Kasuistik 213

Pfortaderthrombosc, Polyzythämia vera, Kasuistik 705

Phäochromozytom, Diagnostik 257

Phäochromozytom, Hypertensive Krise, Therapic 258

Phäochromozytom, maligne Form 257

Phäochromozytom, Pathophysiologie 256

Pharmakokinetik, Schwangerschaft 631

Physikalische Therapie (328)

Phylotherapie, Amara-Pracparationen, Gastrointestinale Dysfunktion 465

Phytotherapic, Anwendungsbereiche 464

Phytotherapic, Bitterstoffe, Gastrointestinalc Dysfunktion 464

Phytotherapie, Chelidonium-Präparationen. Postcholezystektomie-Syndrom 467

Phytotherapie, Gerbstoffdrogen, Diarrhoe 468

Phytotherapie, Gingko biloba-Präparationen. Durchblutungsförderung 470

Phytotherapie, Glykosidtherapie, Herzinsuffizienz 468

Phytotherapic, Immunstimulation 472

Phytotherapic, Ingwer-Präparationen, Dumping-Syndrom 466

Phytotherapie, Laxantien, ObstipationsSyndrom 468

Phytotherapie, Rauwolfia-Präparationen, Kreislaufstörungen 470

Phytotherapie, Wirkungsgemische 463

Pickwick-Syndrom, Pulmonale Zirkulation 681

Piperazin, Teratogene Wirkung, Spalthand, Kasuistik 217

Plazcntalösung, Gerinnungsstörung 613

Plazentapassage, Mechanismen 632

Pneumocystis carinii-Pneumonie, HIV-Infektion 83
Polyarthritis, Crohnsche Krankheit, Kasuistik 285

Polyposis-Syndrome intestinale, Klassifikation 701

Polyzythämia vera, Pfortaderthrombose, Kasuistik 705

Postcheolzystcktomie-Syndrom, Phytotherapie 466

Präeklampsie, HELPP-Syndrom, Gerinnungsstörung 616

Präventivmedizin (322)

Proktologie, Atlas (278)

Proktologie, Lehrbuch (777)

Proktologie praktische (509)

Prolactinom, Diagnostik 43

Prolactinom, Kasuistik 43

Prolactinom, Kernspintomogramm 44

Prolactinom, Therapic 44

Prostaglandine, Embryotoxisches Risiko 202

Prostaglandine, Ulcustherapie 741

Pseudohermaphroditismus, männlicher, Rezeptorstörung 450

Pseudohypoaldosteronismus, Rezeptorstörung 452

Pscudohypoparathyreoidismus, Rezeptorstörung 448

Psychopharmaka (502)

Psychosomatik, Allgemeinmedizin (312)

Pulmonale Hypertonie, akute, Bronchialzirkulation 666

Pulmonale Hypertonie, Atemregulationsstörungen 681

Pulmonale Hypertonie, Bonchitis chronisch obstruktive 671

Pulmonale Hypertonic. Bronchialzirkulation 666

Pulmonale Hypertonic, chronische, Bronchialzirkulation 667

Pulmonale Hypertonic, Gefäßbett, Histologie 676

Pulmonale Hypertonic, Lungenemphysem 673

Pulmonale Hypertonic, Lungenfibrosc 677

Pulmonale Hypertonic, Pathophysiologic 692

Pulmonale Hypertonie, Therapie, Substanzgruppen 691

Pulmonale Hypertonic, Therapieansatz, Grundleiden 689

Pulmonale Hypertonic, Ursachen 688

Pulmonale Hypertonie, Ursachen, Übersicht 681

Pulmonale Zirkulation, Anatomie 653

Pulmonale Zirkulation, Autonome Innervation, Parasympathicus 656

Pulmonale Zirkulation, Autonome Innervation, Sympathicus 655

Pulmonale Zirkulation, Gefäßendothel, Gefäßrelaxierender Faktor 659

Pulmonale Zirkulation, Gefäßrekrutierung 654

Pulmonale Zirkulation, Hypoxie akute, Vasokontriktion 657

Pulmonale Zirkulation, Hypoxie chronische 658

Pulmonale Zirkulation, Körperliche Belastung 657

Pulmonale Zirkulation, Lungenemphysem 673

Pulmonale Zirkulation, Lungenfibrose 676
Pulmonale Zirkulation, Schlafzustand 683 Pulmonale Zirkulation, Vasoaktive Mediatoren, Übersicht 659

Pulmonale Zirkulation, Wachzustand 685

Pulmonale Zirkulation, Zonen-Modell 654

Radiojodtherapie, Indikationsstellung

Radiojodtherapie, Risiken 578

Radiojodtherapie, Therapieziel 577

Respiratorische Therapie (297)

Retinoide, Embryotoxisches Risiko 201

Rezeptor-Krankheiten, Angst-Syndrome 425

Rezeptor-Krankheiten, Asthma bronchiale 438

Rezeptor-Krankheiten, Depressive Syndrome 422

Rezeptor-Krankheiten, Endokrinologie 445

Rezeptor-Krankheiten, Herzinsuffizienz. 407

Rezeptor-Krankheiten, Hypertonie 404

Rezeptor-Krankheiten, Insulinrezeptor-Defekte 390

Rezeptor-Krankheiten, Lipoprotein-Stoffwechsel 372

Rezeptor-Krankheiten, Myasthenia gravis 414

Rezeptor-Krankheiten, Schizophrenie 424

Rezeptor-Krankheiten, Tumorwachstum 430

Rheumatische Erkrankungen

Sarkoidose (455)

Sarkoidose, Diagnostik 352

Sarkoidose, Herztod plötzlicher, Kasuistik 351

Sarkoidose, Klinische Symptomatik 352

Scavenger-Rezeptor, Lipoprotein-Stoffwechsel 381

Schilddrüsencarcinom, Schwangerschalt 624

Schilddrüsencarcinome, C-Zcllcarcinom, Therapic 567

Schilddrüsencarcinome, differenzierte, Therapie 565

Schilddrüsencarcinome, differenzierte, Langzeitbehandlung 583

Schilddrüsencarcinome, D-Zell-Carcinom, Langzeitbchandlung 584

Schilddrüsencarcinome, Maligne Lymphome 568

Schilddrüsencarcinome, Operative Behandlung 574

Schilddrüsencarcinome, Opcrative Therapie 574

Schilddrüsencarcinome, Papilläres Carcinom, Therapie 565

Schilddrüsenerkrankungen, Autonomic, Scintigraphie 544

Schilddrüsenerkrankungen, Carcinome, Diagnostik 564

Schilddrüsenerkrankungen, Carcinome, Therapie 564

Schilddrüsenerkrankungen, Diagnostik, Schilddrüsenhormone 529

Schilddrüsenerkrankungen, Diagnostik, Scintigraphie 541

Schilddrüsenerkrankungen, Diagnostik, Sonographie 545

Schilddrüsenerkrankungen, Hormondiagnostik 529 
Schilddrüsenerkrankungen, Hormondiagnostik, TRH-Test 533

Schilddrüsenerkrankungen, Hormondiagnostik, TSH-Test 523

Schilddrüsenerkrankungen, Immundiagnostik, Antikörper 538

Schilddrüsenerkrankungen, Immundiagnostik, Mikrosomale Antikörper (MAK) 539

Schilddrüsenerkrankungen, Immundiagnostik, Thyreoglobulin 539

Schilddrüsenerkrankungen, Immundiagnostik, Thyreoglobulin-Antikörper (TAK) 539

Schilddrüsenerkrankungen, Immundiagnostik, Thyreotropin-Rezeptor-Antikörper (TRAK) 539

Schilddrüsenerkrankungen, Kalter Knoten 573

Schilddrüsenerkrankungen, Krankheitsbilder, Jodexposition 527

Schilddrüsenerkrankungen, Krankheitsbilder, Krisen 527

Schilddrüsenerkrankungen, Krankheitsbilder, Ophthalmopathie 526

Schilddrüsenerkrankungen, Langzeitbehandlung 580

Schilddrüsenerkrankungen, Morphologische Diagnostik, Stufenprogramm 548

Schilddrüsenerkrankungen, Operative Behandlung 570

Schilddrüsenerkrankungen, Organmanifestationen 524

Schilddrüsenerkrankungen, Organmanifestationen, Energiestoffwechsel 525

Schilddrüsenerkrankungen, Organmanifestationen, Herz-Kreislaufsystem 524

Schilddrüsenerkrankungen. Organmanifestationen, Nervensystem 525

Schilddrüsenerkrankungen, Radiojodtherapie 577

Schilddrüsenerkrankungen, Scintigraphie. Gammakamcra 541

Schilddrüsenerkrankungen, Scintigraphic, Indikationen 542

Schilddrüsenerkrankungen, Scintigraphic. Jod-131 541

Schilddrüsenerkrankungen, Scintigraphie, Technetium 541

Schilddrüsenerkrankungen, Sonographie. Feinnadelpunktion 547

Schilddrüsenerkrankungen, Sonographie, Parenchymveränderungen 546

Schilddrüsenerkrankungen, Sonographie. Schilddrüsenvolumen 546

Schilddrüsenerkrankungen, Sonographic, Technische Voraussetzungen 545

Schilddrüsenerkrankungen, Sonographie, Thyreoiditiden 546

Schilddrüsenerkrankungen, Symptomatologie 523

Schilddrüsenerkrankungen. Therapie, Struma cndemische 550

Schilddrüsenerkrankungen. TSH-Sckrelion, Einfluß von T 3 und T4 5.34

Schilddrüsenhormone, Diagnostik, Einflußfaktoren 529

Schilddrüsenhormone, Diagnostik, moderne Strategie 530

Schilddrüsenhormone, Diagnostik, T4 versus T3 529

Schilddrüsenhormonc, TSH-Sckretion 534
Schilddrüsenhormonresistenz, Rezeptorstörung 449

Schistosomiasis, Endoskopische Diagnostik 811

Schizophrenie, Dopaminerge Neuronensysteme 424

Schlaf-Apnoe, Pulmonale Zirkulation 683

Schulter-Arm-Syndrom (368)

Schutzimpfungen, Tropen (455)

Schwangerschaft, Arzneimittel, Embryotoxisches Risiko 195

Schwangerschaft, Arzneimittel, Embryotoxisches Risiko, Acetylsalicylsäure 195

Schwangerschaft, Arzneimittel, Embryotoxisches Risiko, Piperazin 217

Schwangerschaft, Arzneimittel, Embryotoxisches Risiko, Prostaglandine 202

Schwangerschaft, Arzneimittel, Embryotoxisches Risiko, Retinoide 201

Schwangerschaft, Arzneimittel, Embryotoxisches Risiko, Virustatica 202

Schwangerschaft, Arzneimittel, Embryotoxizität, Analgetica 199

Schwangerschaft, Arzneimittelverordnung 193

Schwangerschaft, Arzneimittelverordnung (629)

Schwangerschaft, Bakteriuric, Bedeutung 604

Schwangerschaft, Blutdruckmessung 602

Schwangerschaft, Diabetes mellitus 606

Schwangerschaft, Diabetes mellitus, Ncugeborenes 610

Schwangerschaft, Diabetes mellitus, Patientenführung, geburtshilfliche 609

Schwangerschaft, Diabetes mellitus, Patientenführung, internistische 608

Schwangerschaft, Diabetes mellitus. Schwangerschaftsabbruch 607

Schwangerschaft. Geburtshilfe, Gerinnungsstörungen 612

Schwangerschaft. Gestationsdiabetes 607

Schwangerschaft, Gynäkologische Carcinome, Verlauf 618

Schwangerschaft, Maligne hämatologische Systemerkrankungen 624

Schwangerschaft, Maligne Tumoren. Therapie und Verlauf 623

Schwangerschaft, Medikamentöse Therapic, Teratogenität 630

Schwangerschaft, Mißbildungsrate, Spontaninzidenz 194

Schwangerschaft, Nierenkrankheiten 601

Schwangerschaft normale, funktionelle Veränderungen 601

Schwangerschaft, Onkologische Probleme 618

Schwangerschaft, Teratogene Medikamente, Mißbildungsentstehung 630

Schwangerschaft, Teratogene Medikamente, Plazentaschranke 632

Schwangerschaft, Therapieprobleme 595

Schwangerschaftshochdruck, Additive Therapic 597

Schwangerschaftshochdruck, Antihypertensive Medikamente 597

Schwangerschaftshochdruck, Medikamentöse Therapie 596

Schwangerschaftshochdruck, Prognoseparameter 597

Schwcigepflicht ärztliche (491)

Scrotonin, Carcinoid-Syndrom 348
Sigmoidoskopie, Parasitendiagnostik 810 Sonographie, Abdominale Diagnostik (733)

Sonographie, Notfallmedizin (322)

Splenomegalie, Osteomyelofibrose, Kasuistik 641

Staphylococcus aureus, Toxisches Schocksyndrom (696)

Steven-Johnson-Syndrom, Ätiologie 358

Stevens-Johnson-Syndrom, Mykoplasma pneumoniae, Kasuistik 356

Stoffwechsel, Psychosomatik (726)

Stoßwellenlithotrypsie, extrakorporale. Cholecystolithiasis 794

Stoßwellenlithotrypsie, extrakorporale, Cholelithiasis 793

Stoßwellenlithotrypsie, extrakorporale, Physikalische Grundlagen 792

Stoßwellenlithotrypsie, extrakorporale, Steinortung 793

Stoßwellenlithotrypsie, extrakorporale, Stoßwellenerzeugung 792

Streptokinase-Therapie, Kurzzeitlyse (670)

Struma diffusa, Therapie 552

Struma endemische, Definition 550

Struma endemische, Epidemiologie 550

Struma endemische, Jodinduzierte Hyperthyreose 551

Struma endemische, Jodmangelbedingungen 550

Struma endemische, Langzeitbehandlung 580

Struma endemische, Therapie, Rezidivprophylaxe 553

Struma endemische, Therapic, Struma diffusa 552

Struma endemische, Therapic, Struma nodosa 553

Struma, Hyperthyreose, Operative Behandlung 571

Struma nodosa, Operative Behandlung 570

Struma nodosa. Therapie 553

Sucrallat, Ulcustherapie 736

Teratologie, experimentelle, Kritische Phase 172

Teratologie, Keimentwicklung, Kritische Phase 170

Teratologic, Kritische Phase, Mißbildungsmanifestation 174

Teratologie, Mißbildungen, Extremitäten. Kritische Phase 173

Teratologie, Mißbildungen, Kritische Phase, Latenz 175

Teratologie, Mißbildungstyp, Kritische Phase, Maskierung 176

Teratologie, Mißbildungstyp, Noxen 177

Teratologie, Schwangerschaftsentwicklung. Mißbildungen 171

Theophylin, Elimination 729

Theophyllin, Absorption 728

Theophyllin, Indikationsstellung, Obstruktive Atemwegserkrankungen 731

Theophyllin, Konzentrationsbestimmung 730

Theophyllin, Pharmakodynamik 728

Theophyllin, Pharmakokinetik 728

Theophyllin. Therapie. Dosisanpassung 730

Theophyllin, Therapie, Nebeneffekte 729 
Theophyllin, Therapic, Obstruktive Atemwegserkrankungen 727

Theophyllin. Wirkungsmechanismus 727

Therapiestudien klinische, Rechtliche Probleme (396)

Therapietreue, Compliance 279

Thrombosetherapic, Thrombozytopenie, Heparin-induzierte 515

Thrombozytopenie, Heparin-induzierte, Kasuistik 515

Thymusdrüse, Myasthenia gravis 417

Thymustumor. Kasuistik 293

Thymustumoren, Klassifikation 296

Thyreoidektomie totale. Substitutionstherapie 562

Thyreoiditis, Langzcitbchandlung 583

Torsade de pointes, Antiarrhythmica 339

Transfusionsmedizin (462)

Trombozyten, Funktionsdiagnostik, klinische Bedeutung (251)

TSH-Resistenz. Rezeptorstörung 447

Tumordiagnostik, Kernspin-Histotomographic, hochauflösende 40

Tumordiagnostik. Kernspin-Spektroskopie, Blutplasma, Protone NMR-Spektrum 35

Tumordiagnostik, Kernspin-Spektroskopie. Blutplasma, Spin-Relaxationszeit 37

Tumor-Handbuch (629)

Tumor-Handbuch. TNM-Klassifikation 528

Tumorkranke terminale, Palliative Behindlung 26

Tumorkranke terminale. Palliativstation 26

Tumorkranke terminale, Palliativstation, Ziclsetzung 27

Tumorkranke terminale. Schmerztherapic 28

Tumorkranke terminale, Schmerzherapie. Neurochirurgic 30

Tumorkranke terminale. Schmerztherapie Neurolytische Nervenblockade 30

Tumorkranke terminale, Schmerztherapie, Opialanalgesie 30

Tumorkranke terminale, Schmerztherapie. Schmerzleitungsmodifikation 29

Tumorlciden, Diätctische Einflüsse 493

Tumornachsorge 1

Tumornachsorge, Datengewinnung 16

Tumornachsorge, Drop-out-Rate 20

Tumornachsorge, Entwicklungstendenzen, Datensysteme 21

Tumornachsorge, Internistische Sicht 22

Tumornachsorge, Internistische Sicht, Beratungsstelle für Ärtze 22

Tumornachsorge, Internistische Sicht. Nachsorgefrequenz. 23
Tumornachsorge, Internistische Sicht, Patientenaufklärung 23

Tumornachsorge, Internistische Sicht, Psychische Betreuung 24

Tumornachsorge, Krebsregister 7

Tumornachsorge, Mamma-Programm 10

Tumornachsorge, Nachsorgeempfehlungen 6

Tumornachsorge, Nachsorgeinhalte 15

Tumornachsorge, Nachsorgeleitstelle 17

Tumornachsorge, Onkologie-Schwester 4

Tumornachsorge, Onkologische Schwerpunktpraxis 4

Tumornachsorge, Selbsthilfegruppen 2

Tumornachsorge, Tumorbasis-Dokumentation 14

Tumornachsorge, Tumortherapie, Nebenwirkungen 23

Tumornachsorge, Tumortherapic, Spätfolgen 23

Tumornachsorge, Zusammenarbeit Klinik Praxis 2

Tumornachsorge, Zusammenarbeit Klinik Praxis, ATO 13

Tumornekrosefaktor, TNF-alpha, Klinische Anwendung 717

Tumorpatient, Enterale Ernährung, Gastrostomiesonde, endoskopisch plazierte 805

Tumorwachstum, menschliche Tumoren. Wachstumsfaktoren 435

Tumorwachstum, Menschliche Tumoren. Wachstumsfaktoren-Rezeptoren 436

Tumorwachstum, Wachstumsfaktoren 431

Tumorwachstum, Wachstumsfaktoren, hämatopoctische 4.35

Tumorwachstum, Wachstumsfaktoren-Rezeptoren 431

Tumorwachstum, Wachstumsfaktoren-Rezeptoren, Insulin-ähnliche 433

Tumorwachstum, Wachstumsfaktoren-Rezeptoren, Thyrosinkinase 432

Tumorzelle, Wachstumsmodell 430

Ulcus duodeni, Campylobacter-pylori-positives, Therapie 751

Ulcus duodeni, Langzeittherapie, Antacida, Rezidivraten 739

Ulcus duodeni, Langzeittherapie, H2-Blokker, Rezidivraten 737

Ulcus duodeni, Langzeittherapie, Pirenzepin, Rezidivraten 739

Ulcus duodeni, Langzeittherapic, Prostaglandin-Derivate, Rezidivraten 741

Ulcus duodeni, Langzeittherapie. Sucralfat, Rezidivraten 740
Ulcus pepticum, H2-Blocker. Therapie (413)

Ulcus ventriculi, Campylobacter-pylori-positives, Therapie 751

Ulcus ventriculi, Langzeittherapic, H2Blocker, Rezidivraten 738

Ulcusalmanach (444)

Ulcuskrankheit, Langzeittherapie 734

Ulcuskrankheit, Langzeittherapie, Ergebnisse 736

Ulcuskrankheit, Rezidiv, Praedisponierende Faktoren 734

Ulcustherapie, Mucosabarriere, Sucralfat 736

Ulcustherapie, Mucosabarricre, Wismut 736

Ulcustherapie, Säurehemmung, Antimuskarinika 735

Ulcustherapic, Säurehemmung, Histamin H2-Antagonisten 734

Ulcustherapie, Säurehemmung, Prostaglandine 735

Ulcustherapie, Säurehemmung, Protonenpumpen-Hemmer 735

Ulcustherapie, Säure-Neutralisation, Antacida 735

Untersuchungstechnik, ärztliche (81)

Urtikaria-Vaskulitis, hypokomplementämische, McDuffie's Syndrom 827

Valproinsäure, Teratogenität 180

Valproinsäure, Teratogenität, Mechanismen 189

Valproinsäure, Teratogenitäı, Strukturspezifität 189

Virusmyokarditis, Kardiomyopathie dilatative 520

Virusstatica, Embryotoxisches Risiko 202

Vitamin B12, Makrobiotische Nahrung 594

Vitamin C, Anti-Carcinogene Wirkung 495

Vitamin-D-Resistenz, Rezeptor-Störung 452

Vitamine (328)

Wachstumshormon-Resistenz, RezeptorStörung 446

Wismut-Enzephalopathie, Ursachen 747

Wismut-Intoxikation, Klinisches Bild 747

Wismutsalze, Anwendungsgebiete 745

Wismutsalze, Wirkungsmechanismen 747

Zieve-Syndrom, Hämolytische Anämie 331

Zytokine, Klinische Anwendung 717

Zytokine, Übersicht 717 


\section{Autorenregister}

Al Karawi, M.A., El Shcikh Mohamed, A.R., Sultan Khuroo, M., Neuhaus. H. 807

Arnold, G., Schüle, B., Morl, H. 697

Autenrieth, G. $\rightarrow$ Baur, X. 347

Autenrieth, G. $\rightarrow$ Krawietz, W. 586

Autenrieth, G. $\rightarrow$ Scheidt. W. von 58

Autenrieth, G. $\rightarrow$ Werdan, K. 710

Bäcker, U. $\rightarrow$ Gathof, B. 124

Baltzer, J. 618

Bamberg, M. $\rightarrow$ Benker, G. 564

Bartl, R. $\rightarrow$ Hoever, C. 641

Baum, H.P. $\rightarrow$ Piper. C. 824

Baumgart. P., Walger, P., Losse. H., Vetter, H. 252

Baur, X., Bortsch, A., Autenrieth, G., Jacob, K., Neudert, J., Schneider, B., Werdan, K. 347

Baur, X. $\rightarrow$ Hcigl, F. 350

Baur, X. $\rightarrow$ Hettich, R. 832

Behr, J., König, G., Reincrtshofer, Th., Lund, R.,

Scheidt, W. von. Fruhmann, G. 828

Benker, G., Reiners, Chr., Krause, U., Bamberg. M., Reinwein, D. 564

Benkert, O. $\rightarrow$ Wetzel, H. 420

Bertsche, O. $\rightarrow$ Kampik, G. 479

Beuckelmann, D., Pritzl, N., Rienmüller, R., Werdan, K. 285

Beuckelmann. D. $\rightarrow$ Krawietz. W. 586

Beuers, U.. Kruis, W., Drasch, G.A., Jacob, K., Pape, G.R. Paumgartner. G. 648

Börner, N. $\rightarrow$ Gräf, P. 778

Bortsch, A. $\rightarrow$ Baur, X. 347

Brabant, G. $\rightarrow$ Mühlen. A. von zur 533

Braun, B. $\rightarrow$ Scheffler, P. 54

Brenner, P. $\rightarrow$ Meyer, H.H. 217

Brodde, O.-E. 397

Brown, K. $\rightarrow$ Scheidt, W. von 58

Brunner, K. $\rightarrow$ Budmiger, H 51

Buchborn, E. 457, 459

Budmiger, H., Bühler, $H$.,

Brunner, K., Siegenthaler-

Zuber, G. 51

Bühler, H. $\rightarrow$ Budmiger, $H$. 51

Castro, L.A. $\rightarrow$ Stoffner-

Hacker, D. 360
Clasen, W. $\rightarrow$ Maurin, N. 354

Classen, M. 787

Classen, M. $\rightarrow$ Schepp, W. 734

Colla, F. $\rightarrow$ Lüthy, R. 82

Daschner. F. 345

Daschner, F., Kappstcin, I. 206

Deinhardt, F. $\rightarrow$ Gathof, B. 124

Diehl, V. $\rightarrow$ Mertens, Th. 131

Dölle, W. 715

Dölle, W. $\rightarrow$ Herken. H. 141

Dralle, H. 570

Drasch, G.A. $\rightarrow$ Beucrs, U. 648

Dreyer, M., Rüdiger, H.W. 390

Eberle, J. $\rightarrow$ Gathol, B. 124

Edel, H.H. 601

Edmonds, D. $\rightarrow$ Vetter, W 224

Egberts, E.-H. $\rightarrow$ Scheurlen, M. 755

Egidy, H. von $\rightarrow$ Piper. C. 824

Ehrhart, E. $\rightarrow$ Klaubert, W. 705

El Sheikh Mohamed, A.R. $\rightarrow$ Al Karawi, M.A. 807

Emrich, D. 541

Engclhardt, D. $\rightarrow$ Hörmann, R. 43

Engelhardt, D. $\rightarrow$ Merk, W. 636

Erdmann, E. $\rightarrow$ Heigl. F. 350

Erdmann, E. $\rightarrow$ Metger, J. 781

Feddersen, C.O. 653

Feuerlein. W. 299, 301

Feuerlein, W. $\rightarrow$ Grünberger, J. 307

Feuerlein. W. $\rightarrow$ Küfner, H. 313

Fink, U. $\rightarrow$ Hörmann, R. 43

Fischer, B. $\rightarrow$ Steppling, H. 671

Friedberg, V. 596

Fruhmann, G. $\rightarrow$ Behr, J. 828

Fruhmann, G. $\rightarrow$ Hettich, R 832

Fruhmann. G. $\rightarrow$ Vogelmeier. C. 638

Gathof, A.G. $\rightarrow$ Gathof, B. 124

Gathor, B., Eberle, J., Bäcker. U., Deinhardt, F., Gathof, A.G. 124

Gebauer, A. $\rightarrow$ Krawietz, W. 586

Gcbhardt, K.-H. 487
Geibel, A. $\rightarrow$ Hohnloser, S.H. 338

Goebel, F.-D. 92

Gokel, J.M. $\rightarrow$ Heigl, F. 350

Görg, C., Görg, K., Pflüger,

K.-H., Havemann, K. 645

Görg, K. $\rightarrow$ Görg, C. 645

Graeff, H. $\rightarrow$ Hugo, R. von 612

Gräf, P., Börner, N.. Reichert. M., Weilemann, L.S., Meyer, J. 778

Greminger, P., Schneider, E., Siegenthaler. W., Vetter, W. 246

Greminger, P. $\rightarrow$ Vetter. W. 224

Gries, F.A. $\rightarrow$ Somville, I. 606

Grob, P.J. 73

Gruenagel, H.H., Krapp. J., Molzahn, E. 13

Grünberger, J., Linzmayer, L. Feucrlein, W. 307

Grundmann, E. 1

Günther, R.W. $\rightarrow$ Merk, W 636

Halerkamp, O. 34

Hagenmüller, F. $\rightarrow$ Hart, R. 815

Hagenmüller, F. $\rightarrow$ Staritz., M. 792

Harloff, M., Schulz. J., Roschke, W.. Riemann, J.F. 589

Hart, R., Hagenmüller, F. 815

Hartmann. A. $\rightarrow$ Kleinschmidt. R. 293

Hartmann, H., Hunsmann, G. 67

Havemann, K. $\rightarrow$ Görg, C. 645

Havemann, K. $\rightarrow$ Kiefer, P. 430

Hehlmann, R. 112

Hehrmann, R. 523

Heigl, F., Steinbeck, G., Baur, X., Erdmann, E., Herbert, M., Gokel, J.M., Remberger, K. 350

Heil, W., Rick, W. 818

Heimpel, H. $\rightarrow$ Schneider, W.A. 46

Hellstern, A. $\rightarrow$ Leuschner, U. 788

Hellstern, P. $\rightarrow$ Scheffler, P. 54

Hepp, H. 595

Herbert, M. $\rightarrow$ Heigl, F. 350

Herken, H., Dölle, W. 141

Hettich, R., Baur, X., Fruhmann, G. 832

Hillebrand, G., Mittermüller, J. 364

Hillebrand, G. $\rightarrow$ StoffnerHacker, D. 360

Hiller, E. $\rightarrow$ Langecker, $P$. 515
Hintze, G. $\rightarrow$ Köbberling, J. 550

Hoever, C., Schalhorn, A.. Wilmanns. W.. Ledderose. G., Bartl. R. 641

Hohnloser, S.H., Meinertz, T. Zehender, M., Geibel, A., Just. H. 338

Holzgreve, H. 232

Hörmann, R., Mann. K.. Schineis, E., Fink. U.. Oeckler, R.. Engelhardt, D. 43

Horster, F.A. 538

Hugo, R. von, Graeff, H. 612

Huhn, D. $\rightarrow$ Vogelmeier. C. 638

Hunsmann, G. $\rightarrow$ Hartmann, H. 67

Jacob, B. $\rightarrow$ Ritter, M.M. 590

Jacob, K. $\rightarrow$ Baur, X. 347

Jacob, K. $\rightarrow$ Beuers, U. 648

Jäger, $\mathrm{H} .97$

Jung, D. $\rightarrow$ Piper, C. 824

Jungi, W.F. 492

Jüngst, D. $\rightarrow$ Ritter, M.M. 590

Jüngst, D. $\rightarrow$ Sauter, G. 288

Just, H. $\rightarrow$ Hohnloser, S.H. 338

Kampik. G., Bertsche. O. 479

Kappstein, I. $\rightarrow$ Daschner, F 206

Kaukcl. I: 676

Khuroo, S. $\rightarrow$ Al Karawi, M 807

Kicler, P.. Havemann, K. 430

Kiehl, R. $\rightarrow$ Scheffler. P. 54

Klaubert, W.. Weber, B., VothKlaubert, B., Mair, W.. Ehrhart, E. Wilmanns, W. 705

Klauss, V. $\rightarrow$ Ritter, M.M. 590

Klein, H.O. 22

Klcinschmidt, R., Robert7. G.-M., Hartmann, A., Vogel J. 293

Köbberling, J., Hintze, G. 550

Kohl, F.V. 438,688

Köhler, U. 703

König, G. $\rightarrow$ Behr, J. 828

König, G. $\rightarrow$ Vogelmeier, C. 638

Korting, H.C. $\rightarrow$ Pauletzki, J. 356

Krapp, J. $\rightarrow$ Gruenagel, H.H 13

Krause, H.-H., Teisinger, $\mathrm{P}$ 291

Krause, U. $\rightarrow$ Benker, G. 564

Krawiet7., W., Ludwig, B., Beuckelmann, D., Saucrbruch, T., Wittc, J., Gcbauer, A., Autenricth, G 586

Kreuzer, E. $\rightarrow$ Mezger, J. 781 
Kreuzer, E. $\rightarrow$ Werdan, K. 710

Kruis. W. $\rightarrow$ Beucrs, U. 648

Kruis, W. $\rightarrow$ Sauter, G. 288

Kublik, A., Wichert, P. von 727

Küfner, H.. Feuerlein, W. 313

Kuhlmann. U., Lanser, K. 664

Kurrle. E. $\rightarrow$ Schneider, W.A. 46

Land. W. $\rightarrow$ Stoffner-Hacker, D. 360

Langecker, P., Ricss, H., Hiller, E., Wilmanns, W. 515

Lanser, K. $\rightarrow$ Kuhlmann, U. 664

Lauchart. W. $\rightarrow$ Wiedmann, K.H. 765

Ledderose, G. $\rightarrow$ Hoever, C. 641

Lehnen, H. $\rightarrow$ Maurin, N. 354

Leuschner. U.. Hellstern, A. 788

Liebau, H. 239

Linzmayer. L. $\rightarrow$ Grünberger. J. 307

Lippert. T.H. 630

Losse. H. $\rightarrow$ Baumgart. P. 252

Ludwig. B. $\rightarrow$ Krawietz. W. 586

Lund. R. $\rightarrow$ Behr. J. 828

Lüscher, T.F. 279

Lüthy. R., Colla, F., Schläpfer. R.. Täuber, M., Siegenthaler. W. 82

Lüthy, R. $\rightarrow$ Siegenthaler, W. 65

Lüthy, R. $\rightarrow$ Vogt, M. 103

Mai. J. $\rightarrow$ Schaberg, T. 510

Mair. W. $\rightarrow$ Klaubert, W. 705

Mann. K. $\rightarrow$ Hörmann, R. 43

Mann. K. $\rightarrow$ Merk, W. 636

Mannes, G.A. $\rightarrow$ Pauletzki, J. 356

Maurin, N., Lehnen, H., Clasen, W.. Sieberth, H.-G. 354

Meinertz, T. $\rightarrow$ Hohnloser, S.H. 338

Menge. H. 745

Merk. W., Mann, K., Günther, R.W., Engelhardt, D. 636

Merker, H.-J. 170

Mertens, Th., Dichl, V. 131

Metz, J. $\rightarrow$ Piper, C. 824

Meyer. H.H., Brenner, P. 217

Meyer. J. $\rightarrow$ Gräf, P. 778

Meyer. P., Wilms, K. 717

Mezger, J., Weinhold, Ch., Kreuzer, E., Erdmann, E. 781

Mittermüller, J. $\rightarrow$ Hillebrand, G. 364

Molzahn, E. $\rightarrow$ Gruenagel, H.H. 13

Morl, H. $\rightarrow$ Arnold, G. 697

Motz, W. $\rightarrow$ Strauer, B.E. 260
Mühlen, A. von zur, Brabant, G. 533

Mühlen, A. von zur, Pfannenstiel, P., Siegenthaler, W. 521

Müller, O.A., Werder, K. von 445

Müller-Höcker, J. $\rightarrow$ Vogelmeier, C. 638

Nau, H. 179

Neubert, D., Stahlmann, R. 193

Neudert, J. $\rightarrow$ Baur, X. 347

Neuhaus, H. 796

Neuhaus, H. $\rightarrow$ Al Karawi, M.A. 807

Oeckler, R. $\rightarrow$ Hörmann, R. 43

Ocrtel, H., Sackmann, M., Zwicbel, F.M. 699

Oertel, R., Vetter, W. 270

Pape, G.R. $\rightarrow$ Beuers, U. 648

Pauletzki, J., Mannes. G.A., Sauerbruch, T., Korting, H.C. 356

Paumgartner, G. $\rightarrow$ Beuers, U. 648

Paumgartner, G. $\rightarrow$ Sauter, G. 288

Pawlowski, B. $\rightarrow$ Somville, I 606

Pfannenstiel. P. 545

Pfannenstiel. P. $\rightarrow$ Mühlen. A. von zur 521

Pflüger, K.-H. $\rightarrow$ Görg, C. 645

Pichlmaier, H., ThielemannJonen, 1., Zech, D. 26

Pickardt, C.R. 555

Piper, C., Jung, D., Metz, J., Baum, H.P., Schmitz, G.A., Egidy, H. von 824

Podszus, T. 681

Pritzl, N. $\rightarrow$ Beuckelmann, D. 285

Reichert, M. $\rightarrow$ Gräf, P. 778

Reiners, Chr. 529

Reiners, Chr. $\rightarrow$ Benker, G. 564

Reinertshofer, Th. $\rightarrow$ Behr, J. 828

Reinwcin, D. $\rightarrow$ Benker, G. 564

Remberger, K. $\rightarrow$ Heigl, F. 350

Resch, K. 160

Rick, W. $\rightarrow$ Heil, W. 818

Riccker, G. 329, 784

Riemann, J.F. $\rightarrow$ Harloff, M. 589

Rienmüller, R. $\rightarrow$ Beuckelmann, D. 285

Riess, H. $\rightarrow$ Langecker, P. 515

Ritter, M.M., Jacob, B. Jüngst, D., Klauss, V. 590

Robert7, G.-M. $\rightarrow$ Kleinschmidt, R. 293
Roschke, W. $\rightarrow$ Harloff, M 589

Rüdiger, H.W. $\rightarrow$ Dreyer, M 390

Sackmann, M. $\rightarrow$ Oertel, H. 699

Sauerbruch, T. $\rightarrow$ Krawietz, W. 586

Sauerbruch, T. $\rightarrow$ Pauletzki, J. 356

Sauter, G., Kruis, W., Jüngst, D., Paumgartner, G. 288

Schaberg, T., Mai, J., Thalmann, U., Scibold, M.

Staib, F. 510

Schalhorn, A. $\rightarrow$ Hoever, C. 641

Scheffler, P., Kichl, R., Braun, B., Hellstern, P., Wenzel, E. 54

Scheidt, W. von, Wacker, R. Brown, K., Autenrieth, G. 58

Scheidt, W. von $\rightarrow$ Bchr, J. 828

Schepp, W., Classen, M. 734

Scheurlen, M., Egberts, E.-H. 755

Schicha. H. 577

Schincis, E. $\rightarrow$ Hörmann, R. 43

Schläpfer, R. $\rightarrow$ Lüthy, R. 82

Schleibner, S. $\rightarrow$ StoffnerHacker, D. 360

Schmitz, G.A. $\rightarrow$ Piper, C. 824

Schneider, B. $\rightarrow$ Baur, X 347

Schneider, E. $\rightarrow$ Greminger, P. 246

Schncider, W.A., Kurrle, E. Heimpel, H. 46

Schneller, W. $\rightarrow$ Vogelmeier, C. 638

Schoop, W. 499

Schülc, B. $\rightarrow$ Arnold, G. 697

Schulz, J. $\rightarrow$ Harloff, M. 589

Schusdziarra, V. 801

Schwabe, U. 147

Scibold, M. $\rightarrow$ Schaberg, T. 510

Scidemann, W. 213

Seitz, H.K., Simanowski, U.A. 317

Sicberth, H.-G. $\rightarrow$ Maurin, N. 354

Siegenthaler, W., Vetter, W. 223

Siegenthaler, W., Täuber, M., Lüthy, R. 65

Sicgenthaler, W. $\rightarrow$ Greminger, P. 246

Siegenthaler, W. $\rightarrow$ Lüthy, R. 82

Siegenthaler, W. $\rightarrow$ Mühlen, A. von zur 521

Siegenthaler, W. $\rightarrow$ Vetter, W. 224

Siegenthaler, W. $\rightarrow$ Vogt, M. 103

Siegenthaler-Zuber, G. $\rightarrow$ Budmiger, $H .51$
Simanowski, U.A. $\rightarrow$ Seitz,

H.K. 317

Somville, I., Pawlowski, B., Gries, F.A. 606

Stahlmann, R. $\rightarrow$ Neubert, D. 193

Staib, F. $\rightarrow$ Schaberg, T. 510

Staritz, M., Hagenmüller, F. 792

Steinbeck, G. $\rightarrow$ Heigl, F. 350

Steinmetz, A., Utermann, G. 372

Steppling, H., Fischer, B. 671

Stoffner-Hacker, D., Castro, L.A., Hillebrand, G., Schleibner, S., Wiebecke, B., Land, W. 360

Strauer, B.E., Motz, W. 260

Sultan Khuroo, M. $\rightarrow$

Al Karawi, M.A. 807

Täuber, M. $\rightarrow$ Lüthy, R. 82

Täuber, M. $\rightarrow$ Siegenthaler, W. 65

Teisinger, P. $\rightarrow$ Krause, H.-H. 291

Thalmann, U. $\rightarrow$ Schaberg, T. 510

Thielemann-Jonen, I. $\rightarrow$ Pichlmaier, H. 26

Toyka. K.V. 414

Unschuld. P.U. 503

Utermann. G. $\rightarrow$ Steinmetz, A. 372

Vetter, H. $\rightarrow$ Baumgart, P. 252

Vetter, H. $\rightarrow$ Vetter, W. 224

Vetter, W., Vetter, H., Edmonds, D., Greminger, P., Siegenthaler, W. 224

Vetter, W. $\rightarrow$ Greminger, P. 246

Vetter, W. $\rightarrow$ Oertel, R. 270

Vetter, W. $\rightarrow$ Sicgenthaler, W. 223

Vogel, J. $\rightarrow$ Kleinschmidı, R. 293

Vogelmeier, C., Huhn, D., König, G., Müller-Höcker, J. Schneller, W., Fruhmann, G. 638

Vogi, M., Lüthy, R., Siegenthaler. W. 103

Voth-Klaubert, B. $\rightarrow$ Klaubert, W. 705

Wacker, R. $\rightarrow$ Scheidt, W. von 58

Wagner, H. 472

Walger, P. $\rightarrow$ Baumgart, P. 252

Weber, B. $\rightarrow$ Klaubert, W. 705

Weber, P. $\rightarrow$ Wiedmann, K.H. 765

Weilemann, L.S. $\rightarrow$ Gräf, P. 778

Weinhold, Ch. $\rightarrow$ Mezger, J. 781

Welz, R. 323 
Wenzel, E. $\rightarrow$ Scheffler, P. 54

Wenzel, K.-W. 559

Werdan, K., Kreuzer, E., Autenrieth, G. 710

Werdan, K. $\rightarrow$ Baur, X. 347

Werdan, K. $\rightarrow$ Beuckelmann, D. 285
Werder, K. von $\rightarrow$ Müller, O.A. 445

Wetzel, H., Benkert, O. 420

Wichert, P. von 371

Wichert, P. von $\rightarrow$ Kublik, A 727

Wiebecke, B. $\rightarrow$ StoffnerHacker, D. 360
Wiedmann, K.H., Wcber, P., Lauchart, W.

$$
765
$$

Wilmanns, W. $\rightarrow$ Hoever, C. 641

Wilmanns, W. $\rightarrow$ Klaubert, W. 705

Wilmanns, W. $\rightarrow$ Langecker P. 515
Wilms, K. $\rightarrow$ Meyer, P. 717 Witte, J. $\rightarrow$ Krawietz. W. 586

Zech, D. $\rightarrow$ Pichlmaier, H. 26 Zehender, M. $\rightarrow$ Hohnloser.

S.H. 338

Ziegler, R. 580

Zimmermann, W. 463

Zwiebel, F.M. $\rightarrow$ Oertel, H. 699

Verantwortlich für den Textteil: Prof. Dr. M. Classen. II. Medizinische Klinik. Klinikum rechts der Isar, Technische Universität München, Ismaninger Straße 80, D-8000 München 80. Verantwortlich für die Rubrik Kasuistik: Prof. Dr. G. Riecker. Direktor der Medizinischen Klinik I des Klinikums Großhadern. Marchioninistr. 15. D-8000 München 70.

Verantwortlich für die Rubrik Klinische Chemie: Prof. Dr. W. Rick. Medizinische Einrichtungen der Universität Düsseldorf. Zentralinstitut für Klinische Chemie und Laboratoriumsdiagnostik, Moorenstr. 5. D-4000 Düsseldorl'1.

Für den Anzeigenteil: E. Lückermann. H. Hüttig. Heidelberger Platy. 3. D-1000 Berlin 33. Springer-Verlag Berlin Heidelberg New York Tokyo.

Druck der Universitätsdruckerei H. Stürt\% AG. D-8700 Wür\%burg. Printed in Germany. r Springer-Verlag Berlin Heidelberg 1988 Springer-Verlag GmbH \& Co. KG. D-1000 Berlin 3.

Dieses Heft enthält eine Beilage der Firmen Lange \& Springer. Berlin/Schwer Verlag. Stuttgart sowie eine eingeklebte Karte des Springer-Verlages Berlin Heidelberg New York London Paris Tokyo Hong Kong 